\title{
Concatenative Complete Complementary Code Division Multiple Access and its Fast Transform based Implementation
}

\author{
Hikaru Mizuyoshi and Chenggao Han
}

\begin{abstract}
Over multipath channels, complete complementary code division multiple access (CC-CDMA) and convolutional spreading code division multiple access (CS-CDMA) provide inter-channel interference (ICI) free transmission with an enhanced spectral efficiency (SE). However, the convolutional spreading (CS) operation of the systems is computationally complex and involves a high peak-to-average power ratio (PAPR). Address such issues, we propose the concatenative complete complementary code division multiple access (CCC-CDMA). Since the CCCCs can be generated from the rows of the WalshHadamard or discrete Fourier transform (DFT) matrices, the CS operation can be implemented using corresponding fast transforms (FTs) to reduce computational complexity. Simulation results shown that enlargement of spreading factor (SF) strengthens the robustness against clipping noise and the binary CCCC generated by Walsh-Hadamard matrix has excellent robustness against Doppler frequency shifts.
\end{abstract}

\section{Index Terms}

CDMA, complete complementary codes, zero correlation zone sequences, CC-CDMA, CS-CDMA

\section{INTRODUCTION}

Up to now, the generational changes of mobile communication technology were mainly characterized by the adopted multiple access (MA) schemes. In the first generation, the users have shared wireless channel resources by analog frequency division MA (FDMA). The digital

H. Mizuyoshi and C. Han was with the Graduate School of Informatics and Engineering, The University of ElectroCommunications, 1-5-1 Chofugaoka, Chofu-shi, Tokyo, 182-8585, Japan, Email: \{mizuyoshi, han.ic\}@uec.ac.jp 
communication techniques were utilized from second generation with time division MA (TDMA), a representative system is the global system for mobile communications (GSM). After then, code division MA (CDMA) technology was adopted for the third generation wireless communication systems. However, long term evolution (LTE), known as 3.9/4 generation mobile communication standard, did not inherit CDMA. Instead, the orthogonal frequency division multiplexing (OFDM) based orthogonal frequency division MA (OFDMA) is adopted for LTE standard [1], [2].

Nevertheless, if we compare the direct spread (DS)-CDMA with OFDMA, DS-CDMA distinguishes users by the previously assigned signals, called spreading sequences, while the indexes of frequency sub-carrier are used for user identification in OFDMA. To be more specific, CDMA multiplies each user's modulated symbols by corresponding spreading sequence while those are multiplied by several sinusoidal waves one-to-one. Thus, if we treat the sampled sinusoidal waves/signals as spreading sequences in a unified manner, the essential differences between DS-CDMA and OFDMA are as the followings:

1) Number of spreading sequences

Since each user transmits modulated symbols over multiple sub-carriers, OFDMA assigns multiple sequences while single sequence is assigned to each user in DS-CDMA.

2) Synchronization

Each OFDMA packet is composed of the synchronously summarized spread signals and the transmitted symbols are detected synchronously at receiver. On the other hand, DSCDMA transmits the spread signals asynchronously, and hence, synchronization is not necessary at both sides.

3) The cyclic prefix scheme

OFDM based system utilizes the cyclic prefix (CP) scheme to convert the aperiodic convolution between the transmitted packet and channel impulse response (CIR) into periodic convolution.

Therefore, we may regard OFDMA as a special CDMA that assigns multiple sinusoidal signals for each user, transmits and receives signals in the synchronous manner, and employs CP scheme.

From the viewpoint of signal design, the performance of CDMA over multipath channel mainly depends on the correlation properties of the employed spreading sequences. The maximum spectral efficiency (SE) is achievable if we use a sequence set (SS) with the ideal correlation properties, that is, the auto-correlation function of each sequence is zero except at zero shift, and the cross-correlation functions of any distinct sequence pair are zero at all shifts. Unfortunately, 
such an SS is non-existence, and instead, the pseudo-random (PR) sequences which have small side-lobes e.g., Gold sequences, Kasami sequences, and maximum length (M) sequences [3], were widely employed for DS-CDMA.

Even the side-lobes of PR sequence is designed as a small value, the non-ideal cross-correlation causes inter-channel interference (ICI) and involves near-far problem. Thus, DS-CDMA is an ICI limited system since we can not increase overall cell capacity by increment of transmission powers and a complex power control unit is essential to combat near-far problem. On the contrary, OFDMA utilizes the sinusoidal waves which have the ideal periodic cross-correlation property and is an ICI-free system.

While the cross-correlation property of the employed spreading sequences is associated with ICI, the auto-correlation property affects on the accuracy of detected symbols over multipath channels. DS-CDMA spreads the modulated symbols by the sequences that have sharp autocorrelation, over a multipath fading channel, it generally attains the full path diversity i.e., the order equal to the number of independent paths. On the other hand, since the periodic autocorrelations of the sinusoidal waves are constant in amplitude at all shifts, over multipath fading channels, the achievable diversity order of the naive OFDM/OFDMA is only 1. Therefore, in the practical uses of OFDM/OFDMA, codings over sub-carriers is usually applied to improve the attainable diversity order [4], [5]. Nevertheless, the obtaining of the full path diversity for OFDM/OFDMA is not a easy task [6].

On the other hand, to realize ICI-free communication system, the signals with the ideal correlation properties are investigated by numerous researchers. Such approaches can be classified into two classes: complete complementary code (CCC) [7], [8] and zero-correlation zone (ZCZ) sequences [9], [10]. The former utilizes multiple sequences to realize the ideal correlation sum while the ideal correlation properties are designed to achieve on the ZCZ in periodic manner for the latter.

The CCC based ICI-free system, called complete complementary coded CDMA (CC-CDMA), is proposed by Suehiro et al. in [11], [12]. In CC-CDMA, each sub-packet spread by different sequence should be passed through individual matched filter. According as the separation of subpackets is done on time or frequency domain, it can be further classified into two categories: time division multiplex (TDM) CC-CDMA [12] and frequency division multiplex (FDM) CC-CDMA [13]. To prevent inter-sub-packet interferences caused by multipath propagation, TDM CCCDMA requires zero padding $(\mathrm{ZP})$ or $\mathrm{CP}$ schemes for each sub-packet, and hence, it is inferior 
to FDM CC-CDMA in term of spectral efficiency (SE) but is superior in system performance and implementation complexity [14]-[16].

The concept of ZCZ sequences has been first appear in [9] and originally applied to realize ICI-free quasi-synchronous (QS)-CDMA which keeps orthogonality between channels if user's time delays are occur within a few chips [17], [18]. After that, the ZCZ sequencers based CDMA employing convolutional spreading (CS) scheme [12] was investigated by Weerashinghe et al. under the name of CS-CDMA [19], [20]. They shown that associate with multiple-input single-output (MISO), CS-CDMA provides the full transmit and path diversities [21]. Later, by utilizing iterative partial multiuser detection (IPMD) to CS-CDMA [22], Yue et al. realized an SE higher than DS-CDMA and chip-interleaved block spread (CIBS)-CDMA proposed in [23].

To achieve a high SE, both CC- and CS-CDMAs utilize the CS scheme which involves high peak-to-average power ratio (PAPR). Thus, Weerasinghe et al. investigated the robustness against clipping noise for various $\mathrm{ZCZ}$ sequences and indicated the $\mathrm{M}-\mathrm{ZCZ}$ sequences constructed from M-sequence is the most robust [24]. The sequence selection also affects the performance of CSCDMA over fast fading channels and the inferior-to-superior relationship is related to the use of the channel estimation at the receiver. Without channel estimation, MM-ZCZ is superior to the ZCZ sequences constructed from Chu sequence [25], noted by Chu-ZCZ, while a elaborately designed channel estimation reverses the superiority [26]-[28].

A difference between CC- and CS-CDMA is that, in CC-CDMA, each user utilizes multiple spreading sequences as well as OFDMA while a single sequence is used in CS-CDMA. Consequently, to prevent inter-sub-packet interferences over multipach channels, CC-CDMA requires multiple CPs/ZPs while single CP is required for each packet in CS-CDMA, and in general, CSCDMA achieves higher SE than CC-CDMA. Moreover, since the larger ZCZ of the employed SS, the higher SE of CS-CDMA, we desire a simple construction of ZCZ sequences with the largest ZCZ.

On the other hand, Han et al. have proposed a special class of $\mathrm{CCC}$, named concatenative CCC (CCCC), and constructed the binary and polyphase CCCCs using Walsh Hadamard (WH) and discrete Fourier transform (DFT) matrices, respectively [29]. Given CCCC, we may construct ZCZ sequences by concatenating the sequences in each complementary code set and elongate ZCZ by padding zeros on each sequence before concatenation. Thus, associate with the CSCDMA scheme, the concatenative complete complementary code division multiple access (CCCCDMA), provides the simple ZP scheme to improve SE. Furthermore, in order to to reduce the 
computational complexity of the CS operation, Han et al. proposed a OFDM like fast Fourier transform (FFT) based implementation structure for the transmitter of CCC-CDMA and showed a trade-off relationship between clipping resistance and computational complexity [30].

In this paper, by introduction of interleaver/deinterleaver components, we present a novel comprehensive FT based transceiver structures of both binary and polyphase CCC-CDMAs. We prove the outputs of the proposed structure are equivalent to that of the CS-CDMA employing the ZCZ sequences constructed from CCCC. The numerical results show that CCC-CDMA enhances the resistance against clipping noise by increment of the employed sequence length i.e.,increment of spreading factor (SF) simply and the binary CCC-CDMA has excellent robustness against Doppler frequency shifts. Comparing with OFDMA, the proposed CCC-CDMA is also a synchronous MA transmission that can be implemented by fast transforms (FTs) but it is superior to OFDMA at the achievable diversity order over multipath fading channel, simple countermeasure against clipping noise, and robustness against frequency shifts.

The remain parts of this paper are organized as follows. In Section II, after the definition of the correlation and correlation sum, we introduce some sequence set/family with ideal correlation (sum). In Section III, we review the conventional CC-CDMA and CS-CDMA briefly. Section IV presents the novel FT based tranceiver structures of CCC-CDMA. The performance of CCCCDMA over Reighy fading multipath channel are analyzed in Section V. The numerical results are shown in Section VI and, finally, we conclude this paper in Section VII.

\section{A. Notations}

A vector is denoted by a bold lowercase letter and is also represented with its entries as $\boldsymbol{v}=$ $\left(v_{n}\right)_{n=0}^{N-1} \cdot \mathbf{0}_{N}$ denotes the all zero vector of length $N$. For given vector $\boldsymbol{v}$, let $\boldsymbol{v}(a: b)=\left(v_{n}\right)_{n=a}^{b}$ be the length- $(b-a+1)$ partial vector of $\boldsymbol{v}$. For simplicity, we identify the vector $\boldsymbol{v}$ and a sequence $v(n)$ satisfying $v(n)=v_{n}$ for $0 \leq n<N$ and $v(n)=0$ otherwise. The concatenation of $M$ vectors $\boldsymbol{v}_{m}, 0 \leq m<M$, is denoted by $\left(\boldsymbol{v}_{0} \boldsymbol{v}_{1} \cdots \boldsymbol{v}_{M-1}\right)$ or $\left(\boldsymbol{v}_{m}\right)_{m=0}^{M-1}$. A matrix is denoted by a bold uppercase letter and an $M \times N$ matrix $\boldsymbol{A}$ with its entries as $\boldsymbol{A}=\left[a_{n}^{(m)}\right]_{m=0, n=0}^{M-1, N-1}$. The $m$ th row and the $n$th column vectors of $\boldsymbol{A}$ are denoted by $\boldsymbol{a}^{m}$ and $\boldsymbol{a}_{n}$, respectively. Moreover, we let $\boldsymbol{A}^{*}$ and $\boldsymbol{A}^{H}$ denote the complex conjugate and Hermitian transpose of $\boldsymbol{A}$, respectively. The determinant and rank of $\boldsymbol{A}$ are denoted by $\operatorname{det}(\boldsymbol{A})$ and $\operatorname{rank}(\boldsymbol{A})$, respectively. Let $\boldsymbol{f}_{N}^{i}$ be the $i$ th row of the $N$-dimensional discrete Fourier transform (DFT) matrix $\boldsymbol{F}_{N}=\left[W_{N}^{m n}\right]_{m=0, n=0}^{N-1, N-1}$ with 
$W_{N}=\exp (-2 j \pi / N)$ and $\boldsymbol{h}_{N}^{i}$ be the $i$ th row of the $N$-dimensional Walsh Hadamard transform (WHT) matrix $\boldsymbol{H}_{N}$, whose recursive generation is given by

$$
\boldsymbol{H}_{2^{m}}=\left[\begin{array}{cc}
\boldsymbol{H}_{2^{m-1}} & \boldsymbol{H}_{2^{m-1}} \\
\boldsymbol{H}_{2^{m-1}} & -\boldsymbol{H}_{2^{m-1}}
\end{array}\right]
$$

for $\boldsymbol{H}_{1}=[1]$. An indexed set is a set of numbered elements and is denoted by outline $\mathbb{S}=$ $\left\{s_{n}\right\}_{n=0}^{N-1}$ while a family $\mathcal{S}$ stands for a collection of sets. $\delta(\tau)$ is Kronecker's delta function and $[x]_{L}$ denotes a nonnegative integer which is less than $L$ and satisfies $\left(x-[x]_{L}\right) \bmod L=0$. For an integer $m, \boldsymbol{m}_{2}$ denotes the binary extension of $m$ and $\boldsymbol{m}_{2} \oplus \boldsymbol{n}_{2}$ denotes the bit-wise exclusive OR logic of two binary vectors $\boldsymbol{m}_{2}$ and $\boldsymbol{n}_{2}$. The expectation operation of a random variable $x$ is denoted as $E\{x\}$.

\section{PRELIMinary}

We call a set $\mathbb{S}=\left\{\boldsymbol{s}_{n}\right\}_{n=0}^{N-1}$ consisting of $N$ length- $L$ sequences an $(N, L)$-sequence set (SS), denoted by $(N, L)-\mathbb{S}$, while a set $\mathcal{S}=\left\{\mathbb{S}^{m}\right\}_{m=0}^{M-1}$ consisting of $M(N, L)$-SSs is called an $(M, N, L)$-sequence family $(\mathrm{SF})$ and denoted by $(M, N, L)-\mathcal{S}$.

For two sequences $s$ of length $L$ and $s^{\prime}$ of length $L^{\prime}$, their aperiodic convolution and aperiodic correlation are defined as

$$
\begin{aligned}
\psi_{\mathrm{A}}\left(\boldsymbol{s}, \boldsymbol{s}^{\prime} ; \tau\right) & :=\sum_{\ell=0}^{L-1} s(\ell) s^{\prime}(\tau-\ell) \\
\phi_{\mathrm{A}}\left(\boldsymbol{s}, \boldsymbol{s}^{\prime} ; \tau\right) & :=\sum_{\ell=0}^{L-1} s(\ell)\left[s^{\prime}(\tau+\ell)\right]^{*}
\end{aligned}
$$

respectively. If $s=s^{\prime}, \phi_{\mathrm{A}}\left(s, s^{\prime} ; \tau\right)$ in (2) is called the auto-correlation of $s$ and denoted as $\phi_{\mathrm{A}}(s ; \tau)$ simply. Otherwise, it is called the cross-correlation between $s$ and $\boldsymbol{s}^{\prime}$. Since $\phi_{\mathrm{A}}\left(s, \boldsymbol{s}^{\prime} ; \tau\right)$ takes non-zero values on the interval $-L^{\prime}<\tau<L$, in order to coordinate with the convolution given in (1), we define two length- $\left(L+L^{\prime}-1\right)$ vectors as

$$
\begin{aligned}
& \boldsymbol{\psi}_{\mathrm{A}}\left(\boldsymbol{s}, \boldsymbol{s}^{\prime}\right):=\left(\psi_{\mathrm{A}}\left(\boldsymbol{s}, \boldsymbol{s}^{\prime} ; \tau\right)\right)_{\tau=0}^{L+L^{\prime}-2} \\
& \underline{\phi}_{\mathrm{A}}\left(\boldsymbol{s}, \boldsymbol{s}^{\prime}\right):=\left(\phi_{\mathrm{A}}\left(\boldsymbol{s}, \boldsymbol{s}^{\prime} ;-\tau\right)\right)_{\tau=-L^{\prime}+1}^{L-1}
\end{aligned}
$$

Notice the $\tau$ th entry of $\underline{\phi}_{\mathrm{A}}\left(\boldsymbol{s}, \boldsymbol{s}^{\prime}\right)$ is given by $\underline{\phi}_{\mathrm{A}}\left(\boldsymbol{s}, \boldsymbol{s}^{\prime} ; \tau\right)=\phi_{\mathrm{A}}\left(\boldsymbol{s}, \boldsymbol{s}^{\prime} ; 1-\tau-L^{\prime}\right)$ and we proved the following equalities in Appendix A.

$$
\begin{gathered}
\psi_{\mathrm{A}}\left(s, \psi_{\mathrm{A}}\left(s^{\prime}, \boldsymbol{s}^{\prime \prime}\right)\right)=\boldsymbol{\psi}_{\mathrm{A}}\left(\psi_{\mathrm{A}}\left(s, s^{\prime}\right), \boldsymbol{s}^{\prime \prime}\right) \\
\psi_{\mathrm{A}}\left(s, \underline{\phi}_{\mathrm{A}}\left(s^{\prime}, \boldsymbol{s}^{\prime \prime}\right)\right)=\underline{\phi}_{\mathrm{A}}\left(\psi_{\mathrm{A}}\left(s, s^{\prime}\right), \boldsymbol{s}^{\prime \prime}\right)
\end{gathered}
$$


For two sequences $s$ and $s^{\prime}$ of length- $L$, the periodic convolution and periodic correlation are defined as

$$
\begin{aligned}
\psi_{\mathrm{P}}\left(\boldsymbol{s}, \boldsymbol{s}^{\prime} ; \tau\right) & :=\sum_{\ell=0}^{L-1} s(\ell) s^{\prime}\left([\tau-\ell]_{L}\right) \\
& =\sum_{\ell=0}^{L-1} s\left([\tau-\ell]_{L}\right) s^{\prime}(\ell) \\
\phi_{\mathrm{P}}\left(\boldsymbol{s}, \boldsymbol{s}^{\prime} ; \tau\right) & :=\sum_{\ell=0}^{L-1} s(\ell)\left[s^{\prime}\left([\ell+\tau]_{L}\right)\right]^{*} \\
& =\sum_{\ell=0}^{L-1} s\left([\ell-\tau]_{L}\right)\left[s^{\prime}(\ell)\right]^{*}
\end{aligned}
$$

respectively. Similar with the aperiodic case, if we define length- $L$ vectors as

$$
\begin{aligned}
& \psi_{\mathrm{P}}\left(\boldsymbol{s}, \boldsymbol{s}^{\prime}\right):=\left(\psi_{\mathrm{P}}\left(\boldsymbol{s}, \boldsymbol{s}^{\prime} ; \tau\right)\right)_{\tau=0}^{L-1} \\
& \underline{\phi}_{\mathrm{P}}\left(\boldsymbol{s}, \boldsymbol{s}^{\prime}\right):=\left(\phi_{\mathrm{P}}\left(\boldsymbol{s}, \boldsymbol{s}^{\prime} ;-\tau\right)\right)_{\tau=0}^{L-1}
\end{aligned}
$$

it is not difficult to see that the following equalities

$$
\begin{aligned}
& \psi_{\mathrm{P}}\left(s, \psi_{\mathrm{P}}\left(s^{\prime}, s^{\prime \prime}\right)\right)=\psi_{\mathrm{P}}\left(\psi_{\mathrm{P}}\left(s, s^{\prime}\right), s^{\prime \prime}\right) \\
& \psi_{\mathrm{P}}\left(s, \underline{\phi}_{\mathrm{P}}\left(s^{\prime}, s^{\prime \prime}\right)\right)=\underline{\phi}_{\mathrm{P}}\left(\psi_{\mathrm{P}}\left(s, s^{\prime}\right), s^{\prime \prime}\right)
\end{aligned}
$$

hold for sequences $s, s^{\prime}$, and $s^{\prime \prime}$ of the same length.

For a length $L$ sequence $s$, let

$$
\mathscr{S}^{e}[\boldsymbol{s}]:=\left(s\left([e+\ell]_{L}\right)\right)_{\ell=0}^{L-1}
$$

be the $e$-shifted sequence of $s$. Then, we have

$$
\begin{aligned}
& \phi_{\mathrm{P}}\left(\mathscr{S}^{e}[\boldsymbol{s}], \mathscr{S}^{e^{\prime}}[\boldsymbol{s}] ; \tau\right) \\
= & \sum_{\ell=0}^{L-1} s\left([e+\ell]_{L}\right) s^{*}\left(\left[e^{\prime}+\tau+\ell\right]_{L}\right) \\
= & \phi_{\mathrm{P}}\left(\boldsymbol{s} ; e^{\prime}-e+\tau\right) .
\end{aligned}
$$

For two SSs, $(N, L)$-S and $\left(N, L^{\prime}\right)$ - $\mathbb{S}^{\prime}$, the aperiodic correlation sum is defined as

$$
\Phi_{\mathrm{A}}\left(\mathbb{S}, \mathbb{S}^{\prime} ; \tau\right):=\sum_{n=0}^{N-1} \phi_{\mathrm{A}}\left(\boldsymbol{s}_{n}, \boldsymbol{s}_{n}^{\prime} ; \tau\right) .
$$

If $\mathbb{S} \neq \mathbb{S}^{\prime}$, it is called the aperiodic auto-correlation sum and denoted by $\Phi_{\mathrm{A}}(\mathbb{S} ; \tau)$. Otherwise, (6) is called the aperiodic cross-correlation sum. 


\section{A. Zero correlation zone (ZCZ)-SS}

Definition 1. A sequence $s$ is called the perfect sequence (PS) if the periodic auto-correlation of $s$ is zero except for zero-shift, i.e.,

$$
\phi_{\mathrm{P}}(s ; \tau)=E_{s} \delta(\tau)
$$

where $E_{\boldsymbol{s}}:=\boldsymbol{s} \boldsymbol{s}^{H}$. From (5), each shifted version of a PS $\mathscr{S}^{e}[\boldsymbol{s}], 0 \leq e<L$, is also PS.

Chu-sequence [25] generated by

$$
s(\ell)= \begin{cases}W_{2 L}^{-Q \ell^{2}} ; & \text { even } L \\ W_{2 L}^{-Q \ell(\ell+1)} ; & \text { odd } L\end{cases}
$$

is a well-known polyphase PS, where $Q$ is an integer relatively prime to $L$ and, in this paper, we let $Q=1$ for simplicity.

On the other hand, let $\boldsymbol{a}$ be the M-sequence of length- $L$, that is, a binary sequence with the periodic auto-correlation function

$$
\phi_{\mathrm{P}}(\boldsymbol{a} ; \tau)= \begin{cases}L ; & \tau=0 \\ -1 ; & \text { otherwise }\end{cases}
$$

Then, the two real-valued sequence generated by

$$
s(\ell)=a(\ell)+\frac{-1 \pm \sqrt{L+1}}{L}
$$

is the PS called the modified maximum-length (MM) sequence [31].

Definition 2. An $(M, L)-\mathbb{S}$ is called a zero correlation zone (ZCZ)-SS, denoted by $(M, L ; Z)$ $\mathrm{ZCZ}$, if the periodic auto-correlation of each sequence is zero for $0<|\tau| \leq Z$ and the periodic cross-correlation between any distinct sequence pair is zero for $|\tau| \leq Z$, i.e.,

$$
\phi_{\mathrm{P}}\left(\boldsymbol{s}^{m}, \boldsymbol{s}^{m^{\prime}} ; \tau\right)=E_{\boldsymbol{s}^{m}} \delta\left(m-m^{\prime} ; \tau\right) \quad|\tau| \leq Z
$$

Each $(M, L ; Z)-Z C Z$ satisfies $Z \leq L / M-1$ [32]. However, the equality may not be achieved for a ZCZ-SS with a small alphabet size and the bound is considered as $Z \leq L / 2 M$ for binary ZCZ-SS.

For a PS $s$ of length- $L$, let $Z=\left\lfloor\frac{L}{M}\right\rfloor-1$, then the SS constructed by

$$
\mathbb{S}^{\prime}=\left\{\mathscr{S}^{m(Z+1)}[\boldsymbol{s}]\right\}_{m=0}^{M-1}
$$


is an $(M, L ; Z)-\mathrm{ZCZ}$.

Example 1. While $Q=1$, the length-8 Chu-sequence generated by (7) is

$$
s=\left(W_{16}^{0}, W_{16}^{15}, W_{16}^{12},-W_{16}^{15}, W_{16}^{0},-W_{16}^{15}, W_{16}^{12}, W_{16}^{15}\right)
$$

For the case of $M=2$, the length of ZCZ is $Z=8 / 2-1=3$, and from (10), the SS $\mathbb{S}^{\prime}=\left\{\mathscr{S}^{0}[\boldsymbol{s}], \mathscr{S}^{4}[\boldsymbol{s}]\right\}$ with sequences

$$
\left\{\begin{array}{l}
\mathscr{S}^{0}[s]=\left(W_{16}^{0}, W_{16}^{15}, W_{16}^{12},-W_{16}^{15}, W_{16}^{0},-W_{16}^{15}, W_{16}^{12}, W_{16}^{15}\right) \\
\mathscr{S}^{4}[s]=\left(W_{16}^{0},-W_{16}^{15}, W_{16}^{12}, W_{16}^{15}, W_{16}^{0}, W_{16}^{15}, W_{16}^{12},-W_{16}^{15}\right)
\end{array}\right.
$$

is $(2,8 ; 3)-\mathrm{ZCZ}$.

On the other hand, from the length-7 M-sequence

$$
\boldsymbol{a}=(--+++-+)
$$

the MM sequence generated by (8) is

$$
\boldsymbol{s}=(-0.74,-0.74,1.26,1.26,1.26,-0.74,1.26) .
$$

Let $M=2$, then we have $Z=2$ for this case and the $\operatorname{SS} \mathbb{S}^{\prime}=\left\{\mathscr{S}^{0}[\boldsymbol{s}], \mathscr{S}^{3}[\boldsymbol{s}]\right\}$ with sequences

$$
\left\{\begin{array}{l}
\mathscr{S}^{0}\left[\boldsymbol{s}^{\prime}\right]=(-0.74,-0.74,1.26,1.26,1.26,-0.74,1.26) \\
\mathscr{S}^{3}\left[\boldsymbol{s}^{\prime}\right]=(1.26,1.26,-0.74,1.26,-0.74,-0.74,1.26)
\end{array}\right.
$$

is $(2,7 ; 2)-Z C Z$.

\section{B. Concatenative Complete Complementary Codes}

Definition 3. An $(N, L)$-SS $\mathbb{C}=\left\{\boldsymbol{c}_{n}\right\}_{n=0}^{N-1}$ is called a complementary SS (CSS), denoted by $(N, L)$-CSS, if the auto-correlation sum of $\mathbb{C}$ is zero except for zero-shift, i.e.,

$$
\Phi_{\mathrm{A}}(\mathbb{C} ; \tau)=E_{\mathbb{C}} \delta(\tau)
$$

where we let $E_{\mathbb{C}}=\sum_{n=0}^{N-1} E_{\boldsymbol{c}_{n}}$. Furthermore, an $(M, N, L)$-SF $\mathcal{C}=\left\{\mathbb{C}^{m}\right\}_{m=0}^{M-1}$ is called a complete complementary code (CCC), denoted by $(M, N, L)$-CCC, if the sequences in each row are CSS and the cross-correlation sum between any two distinct CSSs is zero for all shifts, i.e.,

$$
\Phi_{\mathrm{A}}\left(\mathbb{C}^{m}, \mathbb{C}^{m^{\prime}} ; \tau\right)=N L \delta\left(m-m^{\prime}\right) \delta(\tau) .
$$


For given $(M, N, L)$-CCC $\mathcal{C}=\left\{\mathbb{C}^{m}\right\}_{m=0}^{M-1}$, where $\mathbb{C}^{m}=\left\{\boldsymbol{c}_{n}^{m}\right\}_{n=0}^{N-1}$, if the $(M, N L)$-SS generated by concatenating the sequences in each CSS, i.e.,

$$
\mathbb{S}=\left\{\boldsymbol{s}^{m}\right\}_{m=0}^{M-1}, \quad \boldsymbol{s}^{m}=\left(\boldsymbol{c}_{n}^{m}\right)_{n=0}^{N-1}
$$

is $(M, N L ; Z)$-ZCZ, we call $\mathcal{C}$ Concatenative $\mathrm{CCC}(\mathrm{CCCC})$ and denote as $(M, N, L ; Z)$-CCCC.

In oder to describe the constructions proposed in [29] in a unified manner, we let

$$
\boldsymbol{\Omega}= \begin{cases}\boldsymbol{H}_{N} & \text { for binary } \\ \boldsymbol{F}_{N} & \text { for polyphase }\end{cases}
$$

and $\boldsymbol{\omega}^{k}$ be the $k$ th row of $\Omega$. Now, let $\mathcal{C}=\left\{\left\{\boldsymbol{\omega}^{k}\right\}_{n=0}^{N-1}\right\}_{m=0}^{N-1}$ with the interleavring rule $k=$ $\pi^{(m)}(n)$ be the $(N, N, N)$-SF consisting of the rows of $\Omega$. Then, if the interleaving rule is specified by

$$
k=\pi^{(m)}(n)= \begin{cases}\boldsymbol{m}_{2} \oplus \boldsymbol{n}_{2} & \text { for binary } \\ {[m+n]_{N}} & \text { for polyphase }\end{cases}
$$

the resultant SFs are the binary $(N, N, N ; N / 2)$-CCCC and polyphase $(N, N, N ; N-1)$-CCCC. Notice the interleavin rule given in (13) can be deinterleaved by

$$
n=\bar{\pi}^{(m)}(k)= \begin{cases}\boldsymbol{k}_{2} \oplus \boldsymbol{m}_{2} & \text { for binary } \\ {[k-m]_{N}} & \text { for polyphase }\end{cases}
$$

For the $(N, N, L ; Z)$-CCCC, we have the bound on ZCZ as $Z \leq L-1$ and, similar with the ZCZ sequence, we conjecture the bound $Z \leq L / 2$ for the binary CCCCs. Thus, the proposed constructions are optimal in the sense that the qualities on the (conjectured) bounds hold for both cases. Moreover, CCCCs allow ZP to improve the merit figure.

Theorem 1 ( [29]). For given $(M, N, L ; Z)-\operatorname{CCCC} \mathcal{C}=\left\{\left\{\boldsymbol{c}_{n}^{m}\right\}_{n=0}^{N-1}\right\}_{m=0}^{M-1}$, the SF $\mathcal{C}^{\prime}=\left\{\left\{\boldsymbol{c}_{n}^{\prime m}\right\}_{n=0}^{N-1}\right\}_{m=0}^{M-1}$ terminated with length- $T$ zero vector $\boldsymbol{c}_{n}^{\prime m}=\left(\boldsymbol{c}_{n}^{m}, \mathbf{0}_{T}\right)$, is an $(M, N, L+T ; Z+T)$-CCCC and the corresponding ZCZ-SS is an $(M, N(L+T) ; Z+T)$-ZCZ.

Employing such ZP scheme to the binary $\mathrm{CCCCs}$, the merit figure of the resultant ternary ZCZ-SS is improved as

$$
\eta_{T}=\frac{N+2 T}{2(N+T-1)}
$$

and it is asymptotically optimal in the sense that $\lim _{T \rightarrow \infty} \eta_{T}=1$. 
Example 2. We let $M=N=4$ and consider the binary case with the indexes in the binary forms, i.e., $0=[00]_{2}, 1=[01]_{2}, 2=[10]_{2}, 3=[11]_{2}$. Then, if we carry out the additions of indexes over the extended Galois field $\mathrm{GF}\left(2^{2}\right)$, e.g., $2 \oplus 3=[10]_{2} \oplus[11]_{2}=[01]_{2}=1$, we have

$$
\mathcal{C}=\left\{\begin{array}{llll}
\boldsymbol{h}_{4}^{0} & \boldsymbol{h}_{4}^{1} & \boldsymbol{h}_{4}^{2} & \boldsymbol{h}_{4}^{3} \\
\boldsymbol{h}_{4}^{1} & \boldsymbol{h}_{4}^{0} & \boldsymbol{h}_{4}^{3} & \boldsymbol{h}_{4}^{2} \\
\boldsymbol{h}_{4}^{2} & \boldsymbol{h}_{4}^{3} & \boldsymbol{h}_{4}^{0} & \boldsymbol{h}_{4}^{1} \\
\boldsymbol{h}_{4}^{3} & \boldsymbol{h}_{4}^{2} & \boldsymbol{h}_{4}^{1} & \boldsymbol{h}_{4}^{0}
\end{array}\right\}
$$

while the polyphase CCCC

$$
\mathcal{C}=\left\{\begin{array}{rrrr}
\boldsymbol{f}_{4}^{0} & \boldsymbol{f}_{4}^{1} & \boldsymbol{f}_{4}^{2} & \boldsymbol{f}_{4}^{3} \\
\boldsymbol{f}_{4}^{1} & \boldsymbol{f}_{4}^{2} & \boldsymbol{f}_{4}^{3} & \boldsymbol{f}_{4}^{0} \\
\boldsymbol{f}_{4}^{2} & \boldsymbol{f}_{4}^{3} & \boldsymbol{f}_{4}^{0} & \boldsymbol{f}_{4}^{1} \\
\boldsymbol{f}_{4}^{3} & \boldsymbol{f}_{4}^{0} & \boldsymbol{f}_{4}^{1} & \boldsymbol{f}_{4}^{2}
\end{array}\right\}
$$

can be constructed with the natural addition for indexes. Although the resultant CCCCs have the same length $L=N=4$, the ZCZ of the binary CCCC is $Z=2$ while it is increased to $Z=3$ for the polyphase case. Thus, the SS constructed by (12) is a $(4,16 ; 2)-Z C Z$ with merit figure $\eta=2 / 3$. However, if we terminate $\mathbf{0}_{2}$ for each sequence before concatenation as

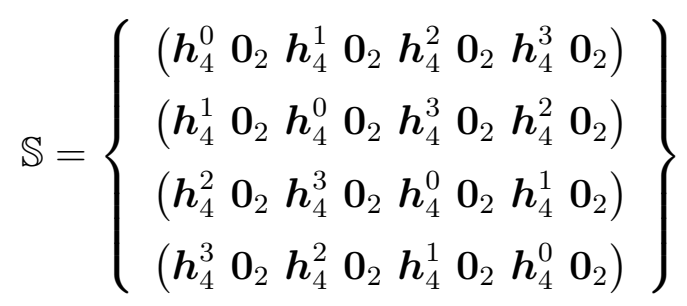

the resultant SS is a $(4,24 ; 4)-\mathrm{ZCZ}$ with improved merit figure $\eta=4 / 5$.

\section{BRIEF REVIEW OF CC-CDMA AND CS-CDMA}

We consider down-link of $M$ users MA systems equipped with single transmitting/receiving antenna. For sake of a unifying description, let $\boldsymbol{u}^{m}, 0 \leq m<M$, be the $m$ th user's modulated symbol vector $^{1}$ of length- $K$ and we assume the antenna transmits the elements of $\overrightarrow{\boldsymbol{x}}$ serially over the $m$ th user's length- $\left(P^{(m)}+1\right)$ quasi-static multipath channel $\boldsymbol{h}_{P}^{m}=\left[h^{(m)}(p)\right]_{p=0}^{P^{(m)}}$ for $E\left\{h^{(m)}(p)\left(h^{(m)}\left(p^{\prime}\right)\right)^{*}\right\}=\sigma_{p}^{2} \delta\left(p-p^{\prime}\right)$. Thus, the received signal is expressed by

$$
\overrightarrow{\boldsymbol{y}}^{m}=\boldsymbol{\psi}_{\mathrm{A}}\left(\boldsymbol{h}_{P}^{m}, \overrightarrow{\boldsymbol{x}}\right)+\overrightarrow{\boldsymbol{\xi}}^{m}
$$

\footnotetext{
${ }^{1}$ Even the lengths of the user's symbol vector are not necessary to be the same in some cases, we assume the same length for simplicity.
} 


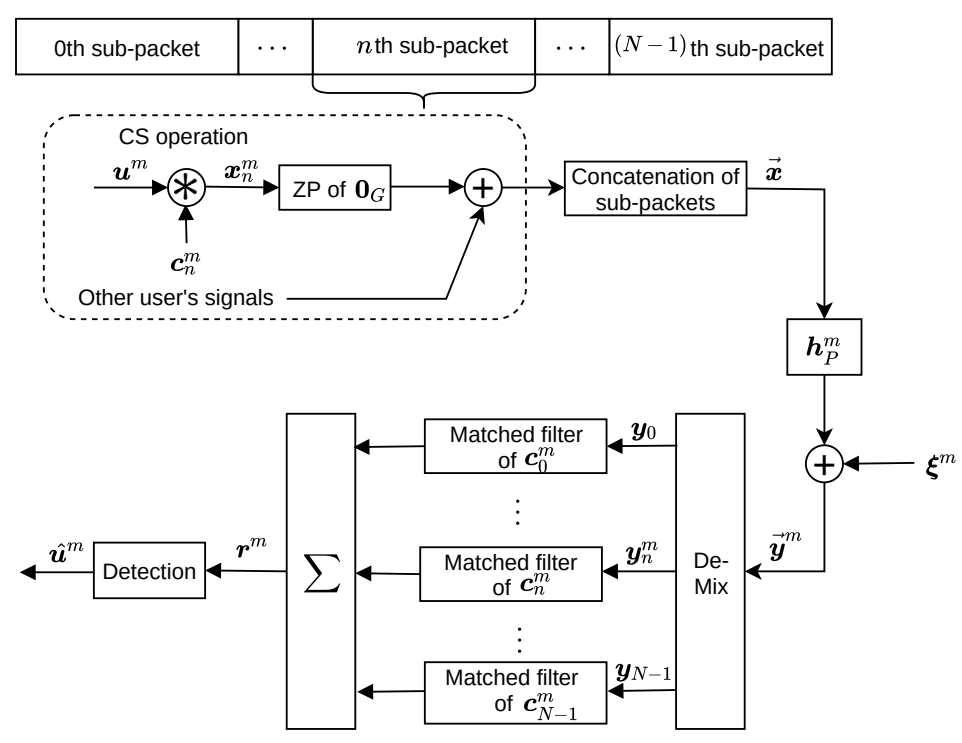

Fig. 1. The down-link system model of CC-CDMA

where $\vec{\xi}^{m}$ is the mutually independent circularly symmetric (CS) additive white Gaussian noise (AWGN) with mean zero and variance $E\left\{\vec{\xi}_{\ell}^{(m)}\left(\vec{\xi}_{\ell^{\prime}}^{(m)}\right)^{*}\right\}=\sigma^{2} \delta\left(\ell-\ell^{\prime}\right)$. In the followings, we let $G \geq \max _{0 \leq m<M}\left\{P^{(m)}\right\}$ and assume the $m$ th user's channel impulse response (CIR) $\boldsymbol{h}_{P}^{m}$ can be perfectly recovered at the $m$ th user's receiver.

\section{A. Review of CC-CDMA [11]}

The tranceiver structure of CC-CDMA is illustrated in Fig. 1. Let $\mathcal{C}=\left\{\mathbb{C}^{m}\right\}_{m=0}^{M-1}$ be an $(M, N, L)$-CCC. In CC-CDMA, the $m$ th CS-SS $\mathbb{C}^{m}=\left\{\boldsymbol{c}_{n}^{m}\right\}_{n=0}^{N-1}$ is assigned as the $m$ th user's spreading SS and all user's modulated symbols are transmitted by packets each of which consisting of $N$ sub-packets. In the $n$th sub-packet, the $m$ th user's modulated symbol vector $\boldsymbol{u}^{m}$ is convolutionally spread by the $n$th spreading sequence $\boldsymbol{c}_{n}^{m}$ to yield the signal-part of the $n$th sub-packet $\boldsymbol{x}_{n}^{m}=\boldsymbol{\psi}_{\mathrm{A}}\left(\boldsymbol{u}^{m}, \boldsymbol{c}_{n}^{m}\right)$. To combat with multipath interference, guard-part $\mathbf{0}_{G}$ is padded on the tail of the signal-part and, by concatenating these sub-packets, we generate $m$ th user's packet $\boldsymbol{x}^{m}=\left(\boldsymbol{x}_{n}^{m} \mathbf{0}_{G}\right)_{n=0}^{N-1}$ of length- $(N(D+L-1))$, where we let $D=K+G$. Finally, all user's packets are summarized together as $\overrightarrow{\boldsymbol{x}}=\sum_{m=0}^{M-1} \boldsymbol{x}^{m}$ and send to the transmitting antenna.

Thanks to the guard-part $\mathbf{0}_{G}$, from the received signal, we can obtain $N$ inter-sub-packet 


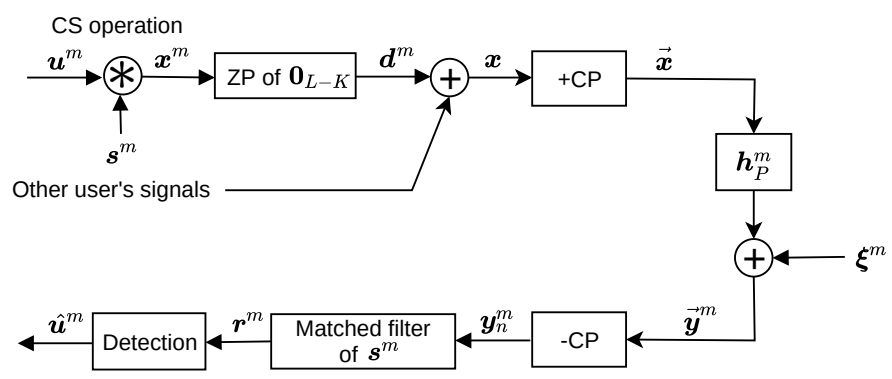

Fig. 2. The down-link system model of CS-CDMA

interference free vectors of length- $\left(K+L+P^{(m)}-1\right)$ as

$$
\begin{aligned}
\boldsymbol{y}_{n}^{m} & =\left(\vec{y}^{(m)}(n(D+L-1)+\ell)\right)_{\ell=0}^{K+L+P^{(m)}-2} \\
& =\boldsymbol{\psi}_{\mathrm{A}}\left(\boldsymbol{h}_{P}^{m}, \boldsymbol{x}_{n}\right)+\boldsymbol{\xi}_{n}^{m}
\end{aligned}
$$

for $0 \leq n<N$, where we let $\boldsymbol{x}_{n}:=\sum_{m=0}^{M-1} \boldsymbol{x}_{n}^{m}$ and $\boldsymbol{\xi}_{n}^{m}:=\left(\vec{\xi}^{(m)}(n(D+L-1)+\ell)\right)_{\ell=0}^{K+L+P^{(m)}-2}$. Each $\boldsymbol{y}_{n}^{m}$ is then inputed into the matched filter of the corresponding spreading sequence $\boldsymbol{c}_{n}^{m}$ and the outputs of matched filter are summarized as

$$
\underline{\boldsymbol{r}}^{m}=\sum_{n=0}^{N-1} \underline{\boldsymbol{\phi}}_{\mathrm{A}}\left(\boldsymbol{y}_{n}^{m}, \boldsymbol{c}_{n}^{m}\right)
$$

We proved in Appendix B that the CC-CDMA provides ICI-free relationship as

$$
\boldsymbol{r}^{m}=E \boldsymbol{\psi}_{\mathrm{A}}\left(\boldsymbol{h}_{P}^{m}, \boldsymbol{u}^{m}\right)+\boldsymbol{\eta}^{m}
$$

where $\boldsymbol{r}^{m}=\left(\underline{r}^{(m)}(\tau)\right)_{\tau=L-1}^{K+P^{(m)}+L-2}, E=L N$, and $\boldsymbol{\eta}^{m}$ is a length- $\left(K+P^{(m)}\right)$ complex Gasussian random vector with mean zero and variance $E\left\{\left(\boldsymbol{\eta}^{m}\right)^{H} \boldsymbol{\eta}^{m}\right\}=E \sigma^{2} \boldsymbol{I}_{K+P^{(m)}}$.

\section{B. Review of CS-CDMA [20]}

Fig. 2 illustrates the downlink transceiver structure of CS-CDMA. In CS-CDMA, the $m$ th sequence of an $(M, L ; Z)$-ZCZ $\mathbb{S}=\left\{\boldsymbol{s}^{m}\right\}_{m=0}^{M-1}$, satisfying $Z \geq K+G-1$, is assigned as the $m$ th user's spreading sequences. At the transmitter, the zero vector $\mathbf{0}_{L-K}$ is padded on the tail of $\boldsymbol{u}^{m}$ to yield the length- $L$ signal vector $\boldsymbol{d}^{m}=\left[\boldsymbol{u}^{m}, \mathbf{0}_{L-K}\right]$. After then, the periodic convolutions $\boldsymbol{x}^{m}=\boldsymbol{\psi}_{\mathrm{P}}\left(\boldsymbol{d}^{m}, \boldsymbol{s}^{m}\right)$ are calculated between $\boldsymbol{d}^{m}$ and $\boldsymbol{s}^{m}$ as CS operation and the resultant signals are summarized with other user's signals as $\boldsymbol{x}=\sum_{m=0}^{M-1} \boldsymbol{x}^{m}$. Finally, after adding length-G cyclic prefix (CP), the signal $\overrightarrow{\boldsymbol{x}}=[\boldsymbol{x}(L-G: L-1), \boldsymbol{x}]$ is sent to the antenna of the transmitter. 
Let $\boldsymbol{y}^{m}$ be the $m$ th user's CP removed signal of length- $L$. Then, as despreading process of $\mathrm{CS}$, we calculate the periodic cross-correlation between the received signal $\boldsymbol{y}$ and the $m$ th user's spreading sequence $s^{m}$ as

$$
\underline{\boldsymbol{r}}^{m}=\underline{\phi}_{\mathrm{P}}\left(\boldsymbol{y}^{m}, \boldsymbol{s}^{m}\right)
$$

and detect the transmitted symbols from the first $K+P^{(m)}$ output $\boldsymbol{r}^{m}=\left(\underline{r}^{(m)}(\tau)\right)_{\tau=0}^{K+P^{(m)}-1}$. In Appendix $\mathrm{C}$, we proved that if we let $E=E_{\boldsymbol{s}^{m}}, \mathrm{CS}-\mathrm{CDMA}$ provides the same relationship given in (16).

\section{The comparison of CC- and CS-CDMAs}

The CC- and CS-CDMAs provide the same ICI-free input-output relationship and achieve higher SE than the DS-CDMAs employing Gold sequence, M-sequence and Walsh sequence [15], [16], [33], [34]. Comparing the SEs of these two systems, CC-CDMA transmits $K M$ symbols using packet of length $N(K+L+G-1)$, where the number of users is bounded by $M \leq N$. Thus, the SE of CC-CDMA is bounded by

$$
\eta_{\mathrm{CC}} \leq \frac{K}{K+G+L_{C}-1}
$$

where $L_{C}$ denotes the length of each sequence.

On the other hand, for CS-CDMA, to transmit $K$ symbols for each user, we have to employ a ZCZ-SS of length $L \geq M(Z+1)$, where $Z \geq K+G-1$. Thus, the length of packet is $L+G \geq M(K+G)+G$ and the SE of CS-CDMA is bounded by

$$
\eta_{\mathrm{CS}} \leq \frac{K}{K+G+G / M}
$$

Comparing (17) with (18), CS-CDMA achieves higher efficiency than CC-CDMA in the case $M\left(L_{C}-1\right)>G$ and vise versa. Additionally, if $K \gg G$, the SE of CC- and CS-CDMAs close to that of OFDMA given by $\eta_{\mathrm{OFDMA}}=K /(K+G)$.

\section{CCC-CDMA WITH ITS IMPLEMENTATIONS}

In this section, we introduce interleaver/deinterleaver components and propose a novel comprehensive FT based implementation structure of CCC-CDMA. 


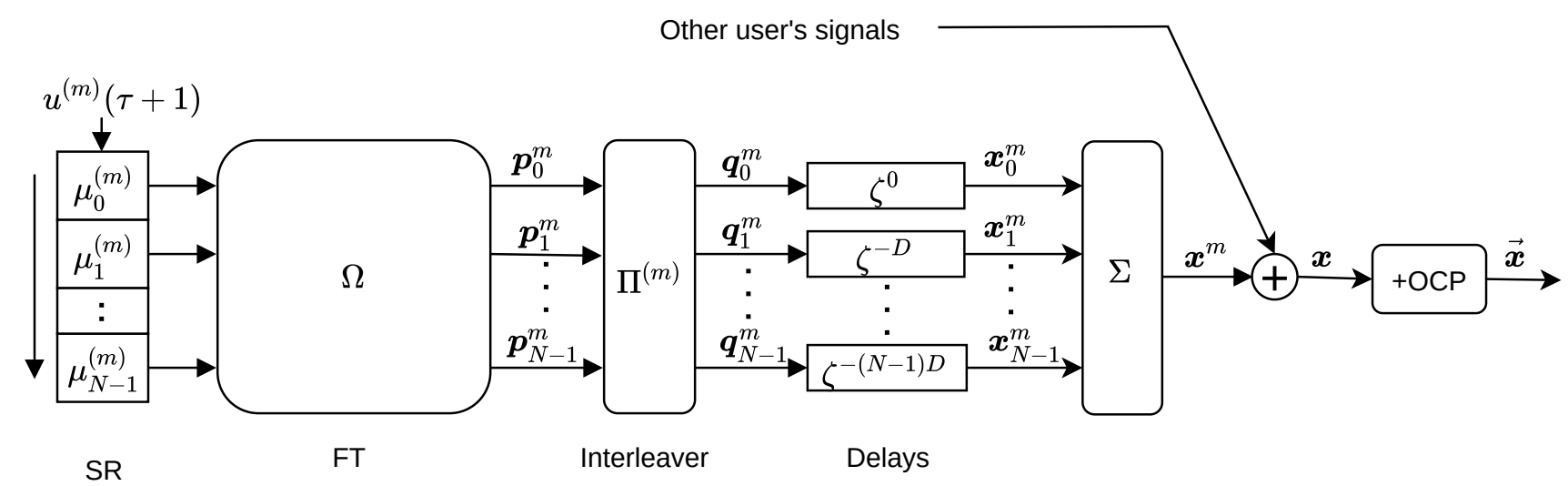

Fig. 3. The FT based transmitter implementation of CCC-CDMA

\section{A. FT based tranceiver structures of CCC-CDMA}

The FT based transmitter structure of CCC-CDMA is illustrated in Fig. 3. Let $\boldsymbol{\mu}^{m}(\tau)$ be the state of the $m$ th user's shift-register (SR) of length $N$ at time $\tau$ and we assume the initial states are set to be $\mathbf{0}_{N}$ for all users. The modulated symbols are serially fed into SR and, after each shift, the $N$-points FT is performed over SR to yield signal $\boldsymbol{p}^{m}(\tau)=\boldsymbol{\mu}^{m}(\tau) \boldsymbol{\Omega}$ until all modulated symbols are shifted out from the SR. Thus, the transmitter performs totally $K+N-1$ FTs, that begins and terminates at the states $\boldsymbol{\mu}^{m}(0)=\left(u^{(m)}(0), \mathbf{0}_{N-1}\right)$ and $\boldsymbol{\mu}^{m}(K+N-1)=\left(\mathbf{0}_{N-1}, u^{(m)}(K-1)\right)$, respectively.

Let $p_{k}^{(m)}(\tau)$ be the $k$ th output of FT at time $\tau$ and let $\boldsymbol{p}_{k}^{m}:=\left(p_{k}^{(m)}(\tau)\right)_{\tau=0}^{K+N-1}$. With the $m$ th user's interleaver $\Pi^{(m)}$ whose interleaving rule $n=\pi^{(m)}(k)$ is specified by (14), $\boldsymbol{q}_{n}^{m}$ is inputed into the delay $\zeta^{-n D}$ to yield $\boldsymbol{x}_{n}=\left(\mathbf{0}_{n D}, \boldsymbol{q}_{n}, \mathbf{0}_{(N-n-1) D}\right)_{n=0}^{N-1}$ and the delayed signals are summarized altogether to yield the $m$ th user's signal vector $\boldsymbol{x}^{m}=\left(\boldsymbol{x}_{n}^{m}\right)_{n=0}^{N-1}$. The resultant signal is added with other user's signals as $\boldsymbol{x}=\sum_{m=0}^{M-1} \boldsymbol{x}^{m}$. Finally, the length- $D$ vector $\boldsymbol{x}(L-G$ : $K+L-1)$, called the overlapped $C P(\mathrm{OCP})$, is appended to the head of signal $\boldsymbol{x}$ to yield the signal $\overrightarrow{\boldsymbol{x}}$, where the length- $K$ signal $\boldsymbol{x}(L: K+L-1)$ is removed from the tail and summarized with $\boldsymbol{x}(0: K-1)$ while the length- $G$ signal $\boldsymbol{x}(L-G: L-1)$ is copied to the head of $\boldsymbol{x}$ as illustrated in Fig. 4.

At the $m$ th user's receiver, after $\mathrm{CP}$ removed, the length- $N D$ vector $\boldsymbol{y}^{m}$ is arranged into a cyclic shift-register (CSR) whose $t$ th value at time $\tau$ can be expressed by

$$
\nu_{t}^{(m)}(\tau)=y^{(m)}\left([t-\tau]_{N D}\right)
$$




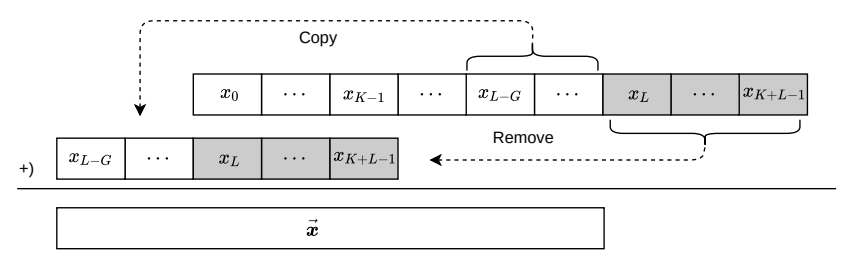

Fig. 4. The operation of OCP

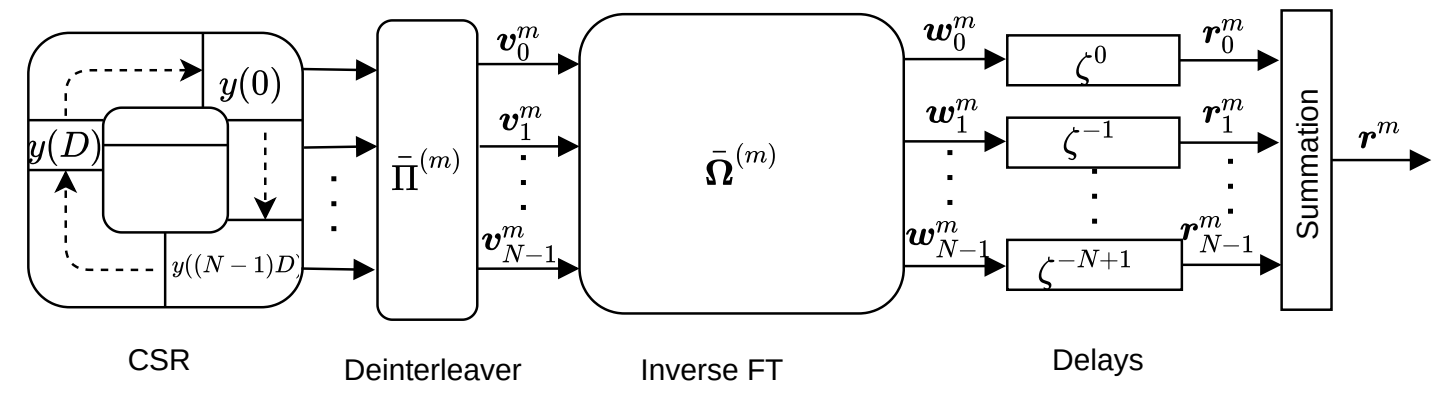

Fig. 5. The FT based receiver implementation of CCC-CDMA

The $D$ spaced $\operatorname{CSR} \nu_{n D}^{(m)}(\tau), 0 \leq d<N$, are connected to the deinterleaver $\bar{\Pi}^{(m)}$ and the $n D$ th $\operatorname{CSR} \nu_{n D}^{(m)}(\tau)$ is inputed into the $k$ th input of the FT $v_{k}^{(m)}(\tau)$ with the deinterleaving rule $k=\pi^{(m)}(n)$ specified by (14). Let $\boldsymbol{v}^{m}(\tau):=\left(v_{k}^{(m)}(\tau)\right)_{k=0}^{N-1}$. Then, the $N$ points transform is performed over the outputs of the deinterleaver as

$$
\boldsymbol{w}^{m}(\tau)=\boldsymbol{v}^{m}(\tau) \overline{\boldsymbol{\Omega}}
$$

and the resultant signal is inputed to delay $\zeta^{-n}$ to yield $\boldsymbol{r}_{n}^{m}=\left(\mathbf{0}_{n}, \boldsymbol{w}_{n}^{m}, \mathbf{0}_{N-n-1}\right)_{n=0}^{N-1}$. Finally, we detect the transmitted data based on the summarized vector

$$
\boldsymbol{r}^{m}=\sum_{n=0}^{N-1} \boldsymbol{r}_{n}^{m}
$$




\section{B. The proof of equivalence}

While the ZCZ-SS generated from CCCC is employed for CS-CDMA, we proved in Appendix $\mathrm{D}$ that the $m$ th user's outputs at transmitter and receiver are represented by

$$
\begin{aligned}
x_{\mathrm{CS}}^{(m)}(\tau) & =\sum_{n=0}^{N-1} \sum_{\ell=0}^{N-1} u^{(m)}\left([\tau-\ell-n D]_{N D}\right) c_{n}^{(m)}(\ell) \\
\underline{r}_{\mathrm{CS}}^{(m)}(\tau) & =\sum_{\ell=0}^{L-1} \sum_{n=0}^{N-1} y^{(m)}\left([-\tau-\ell-n D]_{N D}\right)\left[c_{n}^{(m)}(\ell)\right]^{*}
\end{aligned}
$$

respectively.

In the FT based transmitter structure, on the other hand, since the value of the $\ell$ th SR at time $\tau$ is given by $\mu_{\ell}^{(m)}(\tau)=u^{(m)}(\tau-\ell)$, the $k$ th output of the FT can be expressed as

$$
\begin{aligned}
p_{k}^{(m)}(\tau) & =\sum_{\ell=0}^{N-1} \mu_{\ell}^{(m)}(\tau) \omega_{k}^{(\ell)} \\
& =\sum_{\ell=0}^{N-1} u^{(m)}(\tau-\ell) \omega_{k}^{(\ell)}
\end{aligned}
$$

while the $m$ th user's signal at time $\tau$ is represented by

$$
x^{(m)}(\tau)=\sum_{n=0}^{N-1} x_{n}^{(m)}(\tau)=\sum_{n=0}^{N-1} q_{n}^{(m)}(\tau-n D)
$$

Since the $n$th input of delay are connected with the $k$ th output of FT by interleaving rule $k=\pi^{(m)}(n)$, substituting (21) into (22), we have

$$
\begin{aligned}
x^{(m)}(\tau) & =\sum_{n=0}^{N-1} p_{\pi^{(m)}(n)}^{(m)}(\tau-n D) \\
& =\sum_{n=0}^{N-1} \sum_{\ell=0}^{N-1} u^{(m)}(\tau-\ell-n D) \omega_{\pi^{(m)}(n)}^{(\ell)} \\
& =\sum_{n=0}^{N-1} \sum_{\ell=0}^{N-1} u^{(m)}(\tau-\ell-n D) \omega_{\ell}^{\left(\pi^{(m)}(n)\right)}
\end{aligned}
$$

where the last equality is caused by the symmetry of $\Omega$. Thus, if the interleaving rule is specified by (13), the $m$ th user's output is expressed as

$$
x^{(m)}(\tau)=\sum_{n=0}^{N-1} \sum_{\ell=0}^{N-1} u^{(m)}(\tau-\ell-n D) c_{n}^{(m)}(\ell)
$$


Let $\tau=n^{\prime} D+\ell^{\prime}$ for $0 \leq n^{\prime}<N$ and $0 \leq \ell^{\prime}<D$. Then, since $u^{(m)}(\tau)=0$ if $\tau<0$ or $\tau \geq K$, (23) can be rewritten for the case $n^{\prime}>0$ as

$$
\begin{aligned}
x_{\mathrm{CS}}^{(m)}(\tau) & =\sum_{n=0}^{N-1} \sum_{\ell=0}^{N-1} u^{(m)}\left(\left[\left(n^{\prime}-n\right) D+\left(\ell^{\prime}-\ell\right)\right]_{N D}\right) c_{n}^{(m)}(\ell) \\
& =\sum_{\ell=0}^{\ell^{\prime}} u^{(m)}\left(\ell^{\prime}-\ell\right) c_{n^{\prime}}^{(m)}(\ell)+\sum_{\ell=\ell^{\prime}}^{N-1} u^{(m)}\left(D+\ell^{\prime}-\ell\right) c_{n^{\prime}-1}^{(m)}(\ell) \\
& =x^{(m)}(\tau)
\end{aligned}
$$

and for the case $n^{\prime}=0$ as

$$
\begin{aligned}
x_{\mathrm{CS}}^{(m)}(\tau) & =\sum_{\ell=0}^{\ell^{\prime}} u^{(m)}\left(\ell^{\prime}-\ell\right) c_{0}^{(m)}(\ell)+\sum_{\ell=\ell^{\prime}}^{N-1} u^{(m)}\left(D+\ell^{\prime}-\ell\right) c_{N-1}^{(m)}(\ell) \\
& =x^{(m)}(\tau)+x^{(m)}(L+\tau)
\end{aligned}
$$

Thus, it is not difficult to see that in Fig. 4, the summation of the overlapped part realize (25) while (24) guarantees the copied part consists the CP of CS-CDMA. Moreover, since it is common for all users, we may process the OCP after the summation of all user's signals.

In the proposed receiver structure, since the $k$ th output of the interleaver at time $\tau$ can be expressed as $v_{k}^{(m)}(\tau)=y^{(m)}\left([n D-\tau]_{N D}\right)$, for $n=\bar{\pi}^{(m)}(k)$, the $\ell$ th output of the FT is given by

$$
\begin{aligned}
w_{\ell}^{(m)}(\tau) & =\sum_{k=0}^{N-1} v_{k}^{(m)}(\tau) \bar{\omega}_{\ell}^{(k)} \\
& =\sum_{n=0}^{N-1} y^{(m)}\left([n D-\tau]_{N D}\right) \bar{\omega}_{\ell}^{\left(\bar{\pi}^{(m)}(n)\right)}
\end{aligned}
$$

and, from $r_{\ell}^{(m)}(\tau)=w_{\ell}^{(m)}(\tau-\ell)$, we have

$$
\begin{aligned}
r^{(m)}(\tau) & =\sum_{\ell=0}^{N-1} w_{\ell}^{(m)}(\tau-\ell) \\
& =\sum_{n=0}^{N-1} \sum_{\ell=0}^{N-1} y^{(m)}\left([n D-\tau-\ell]_{N D}\right) \bar{\omega}_{\ell}^{\left(\bar{\pi}^{(m)}(n)\right)} \\
& =\sum_{n=0}^{N-1} \sum_{\ell=0}^{N-1} y^{(m)}\left([-\tau-\ell-n D]_{N D}\right) \bar{\omega}_{\ell}^{\left(\bar{\pi}^{(m)}(N-n)\right)}
\end{aligned}
$$


Now, let $\bar{\Omega}=\left[h_{\ell}^{\left([N-k]_{N}\right)}\right]_{k=0, \ell=0}^{N-1, N-1}$ or $\boldsymbol{F}_{N}^{-1}$ for binary or polyphase cases, respectively. Then, we have

$$
\bar{\omega}_{\ell}^{\left(\bar{\pi}^{(m)}(N-n)\right)}= \begin{cases}h_{\ell}^{\left(\boldsymbol{m}_{2} \oplus \boldsymbol{n}_{2}\right)}=c_{n}^{(m)}(\ell) & \text { for binary } \\ W_{N}^{-(m+n) \ell}=\left[c_{n}^{(m)}(\ell)\right]^{*} & \text { for polyphase }\end{cases}
$$

and the output of (26) coincides with (20) since all elements of the binary CCCC are real value.

\section{PERformance ANALYsis of CCC-CDMA}

In this section, we consider the computational complexity of the proposed structure and evaluate the pair-wise error probability (PEP) for the systems with the input-output relationship given in (16). We assume the maximum-likelihood (ML) detection is employed at the receiver and, for sake of notational simplicity, in the followings, we omit the user index $m$.

\section{A. Complexity}

We consider the computational complexity in terms of the complex multiplications required for CS operation. While an $(N, N, N ; Z)$-CCCC is employed in the CCC-CDMA, the conventional CS-CDMA requires complex multiplications of order $\mathcal{O}\left(N^{2}\right)$ for spreading a modulated symbol. In the proposed FT based structure, on the other hand, since the size- $N$ FFT is performed $(K+N-1) \approx K+N$ times, the number of multiplications required for spreading a modulated symbol is order of $\mathcal{O}\left(\frac{(K+N) N}{K} \log _{2} N\right)$. Thus, for the case $K \gg N$, the polyphase CCC-CDMA has a complexity of order $\mathcal{O}\left(N \log _{2} N\right)$, less than that of $\operatorname{OFDM}(\mathrm{A}) \mathcal{O}\left(\log _{2} K\right)$. Moreover, the binary CCC-CDMA completely eliminates the multiplications in CS operation at the expense of SE.

\section{B. Conditional PEP for ML detection}

We consider the PEP that the transmitted symbol vector $\boldsymbol{u}$ is erroneously detected into $\hat{\boldsymbol{u}}$. Then, the conditional PEP is upper bounded as

$$
\begin{aligned}
P\left(\boldsymbol{u} \rightarrow \hat{\boldsymbol{u}} \mid \boldsymbol{h}_{P}\right) & \leq \operatorname{Pr}\left\{\left\|\boldsymbol{r}-E \boldsymbol{\psi}_{\mathrm{A}}\left(\boldsymbol{h}_{P}, \boldsymbol{u}\right)\right\|^{2} \geq\left\|\boldsymbol{r}-E \boldsymbol{\psi}_{\mathrm{A}}\left(\boldsymbol{h}_{P}, \hat{\boldsymbol{u}}\right)\right\|^{2} \mid \boldsymbol{h}_{P}\right\} \\
& =\operatorname{Pr}\left\{2 \Re\left\{\boldsymbol{e} \boldsymbol{H} \boldsymbol{\eta}^{H}\right\} \leq-\|\boldsymbol{e} \boldsymbol{H}\|^{2} \mid \boldsymbol{h}_{P}\right\}
\end{aligned}
$$

where we let $\boldsymbol{e}:=\boldsymbol{u}-\hat{\boldsymbol{u}}$ and $\boldsymbol{H}$ is the Teoplitz matrix of size $K \times(K+P)$ with the first row and column vectors $\boldsymbol{h}_{0}=\left[\begin{array}{lll}\boldsymbol{h}_{P} & \mathbf{0}_{K-1}\end{array}\right]$ and $\boldsymbol{h}^{0}=\left[h_{0} \mathbf{0}_{K-1}\right]$, respectively. 
Since $\boldsymbol{\eta}$ in (16) is composed of the CS Gaussian random variables with mean zero and variance $N_{0}, \boldsymbol{H} \boldsymbol{\eta}^{H}$ is also a CS Gaussian vector with mean zero and covariance matrix $E\left\{\boldsymbol{H} \boldsymbol{\eta}^{H} \boldsymbol{\eta} \boldsymbol{H}^{H}\right\}=$ $N_{0} \boldsymbol{I}_{K}$. Consequently, $\Re\left\{\boldsymbol{e} \boldsymbol{H} \boldsymbol{\eta}^{H}\right\}$ has the Gaussian distribution with mean zero and variance

$$
D\left(\Re\left\{\boldsymbol{e} \boldsymbol{H} \boldsymbol{\eta}^{H}\right\}\right)=\frac{N_{0}}{2}\|\boldsymbol{e} \boldsymbol{H}\|^{2}
$$

and the conditional PEP is upper-bounded as

$$
P\left(\boldsymbol{e} \mid \boldsymbol{h}_{P}\right) \leq \mathcal{Q}\left(\sqrt{\frac{\|\boldsymbol{e} \boldsymbol{H}\|^{2}}{2 N_{0}}}\right)=\mathcal{Q}\left(\sqrt{\frac{\left\|\boldsymbol{h}_{P} \boldsymbol{E}\right\|^{2}}{2 N_{0}}}\right)
$$

where $\mathcal{Q}(x)$ is the Gaussian $Q$-function and $\boldsymbol{E}$ is the Teoplitz matrix of size $P \times(K+P)$ with the first row and column vectors given by $\boldsymbol{e}_{0}=\left[\begin{array}{ll}\boldsymbol{e} & \mathbf{0}_{P}\end{array}\right]$ and $\boldsymbol{e}^{0}=\left[\begin{array}{ll}e_{0} & \mathbf{0}_{P}\end{array}\right]$, respectively.

\section{PEP over fading channels}

From Craig's representation [35] (also see [36]),

$$
\mathcal{Q}(x)=\frac{1}{\pi} \int_{0}^{\frac{\pi}{2}} \exp \left\{-\frac{x^{2}}{2 \sin ^{2} \alpha}\right\} d \alpha
$$

the bound (27) is written as

$$
P\left(\boldsymbol{e} \mid \boldsymbol{h}_{P}\right) \leq \frac{1}{\pi} \int_{0}^{\frac{\pi}{2}} \exp \left\{-\frac{\left\|\boldsymbol{h}_{P} \boldsymbol{E}\right\|^{2}}{4 N_{0} \sin ^{2} \alpha}\right\} d \alpha
$$

Since the channel is assumed to subject quasi-static uncorrelated multipath Rayleigh fading, $\boldsymbol{h}_{P}$ is consisting of mutually independent Gaussian random variable with mean zero and variance $E\left\{\boldsymbol{h}_{P}^{H} \boldsymbol{h}_{P}\right\}=\boldsymbol{\Sigma}_{P}:=\operatorname{diag}\left[\sigma_{0}^{2}, \sigma_{1}^{2}, \cdots, \sigma_{P}^{2}\right]$, where we let $\sigma_{p}>0, p=0,1, \cdots, P$, for the sake of simplicity.

Then, since $\boldsymbol{h}_{P}$ has the probability density function (PDF) given by

$$
f_{\boldsymbol{h}_{P}}(\boldsymbol{x})=\frac{\exp \left(-\boldsymbol{x} \boldsymbol{\Sigma}_{P}^{-1} \boldsymbol{x}^{H}\right)}{\pi^{P+1} \operatorname{det}\left(\boldsymbol{\Sigma}_{P}\right)}
$$

and the (unconditional) PEP is bounded by

$$
\begin{aligned}
P\left(\boldsymbol{e}^{m}\right) & =\int P\left(\boldsymbol{e}^{m} \mid \boldsymbol{x}\right) f_{\boldsymbol{h}_{P}}(\boldsymbol{x}) d \boldsymbol{x} \\
& \leq \frac{1}{\pi} \int_{0}^{\frac{\pi}{2}} \frac{\left(4 N_{0}\right)^{P+1} \sin ^{2(P+1)} \alpha}{\operatorname{det}\left(\boldsymbol{B} \boldsymbol{\Sigma}_{P}\right)} d \alpha
\end{aligned}
$$

where

$$
\boldsymbol{B}=\|\boldsymbol{E}\|^{2}+4 N_{0}\left(\sin ^{2} \alpha\right) \boldsymbol{\Sigma}_{P}^{-1}
$$


Since $\Sigma_{P}$ is a nonnegative diagonal matrix and

$$
\|\boldsymbol{E}\|^{2}=\left[\phi_{A}(\boldsymbol{e}, i-j)\right]_{i=0, j=0}^{P, P}
$$

is the auto-correlation matrix of $e$ which is a nonnegative definite Hermitian matrix. Thus, we have $\operatorname{det}(\boldsymbol{B}) \geq \operatorname{det}\left(\|\boldsymbol{E}\|^{2}\right)$, and then the PEP is bounded as

$$
P_{n}(\boldsymbol{e}) \leq \frac{C(P+1)}{\operatorname{det}\left(\|\boldsymbol{E}\|^{2}\right)}
$$

where for a positive integer $x$, we let

$$
C(x)=\frac{\left(4 N_{0}\right)^{x}}{\pi \operatorname{det}\left(\Sigma_{x}\right)} \int_{0}^{\pi / 2} \sin ^{2 x} \alpha d \alpha=\frac{2 x-1 C_{x-1} N_{0}^{x}}{\operatorname{det}\left(\boldsymbol{\Sigma}_{x}\right)}
$$

for the binomial coefficient ${ }_{n} C_{r}$. The right-hand side of the bound (32) is finite if and only if $\operatorname{det}\left(\|\boldsymbol{E}\|^{2}\right) \neq 0$, that is, $\operatorname{rank}\left(\|\boldsymbol{E}\|^{2}\right)=P+1$. Thus, if the input-output relationship is given by (16), over the length- $(P+1)$ multipath Reyleigh fading channel, such systems provide the full path diversity of order $P+1$ if $K>P$.

\section{Simulation Results}

We have shown in the previous section that, for the systems which provide the input-output relationship shown in (16), its performance over Rayleigh fading multipath channel does not depend on the sequence selection. Thus, in this paper, we evaluate the resistances against clipping noise and Doppler frequency shifts for the binary and polyphase CCCCs, Chu-ZCZ, and M-ZCZ. For CCCCs, the resistance against clipping noise is known to be monotonically weaken with the increment of padding zeros [30] and, in this paper, we only test the crude CCCCs.

The evaluations are carried out by computer simulations over four paths $(P=3)$ Rayleigh fading channel with uniformly distributed power delay profile (PDP) for the CCCC, Chu-ZCZ, and OFDMA of lengths $L=256 / 1024$ while $L=255 / 1023$ for M-ZCZ. For all cases, we assumed $M=N$ users transmit QPSK modulated symbols of the same length- $(N / 2-P+1)$ per packet, the maximum length of the binary CCCC which provides the lowest SE. Thus, for the CCCCs as well as Chu-ZCZ and OFDMA, $M=N=\sqrt{L}=16$ and 32 for $L=256$ and 1024, respectively, while the lengths of the corresponding QPSK symbol vectors are 6 and 14, respectively, and these are 1 symbol short for M-ZCZ. At the receiver, the ML with Viterbi algorithm is utilized to recover the transmitted symbols and we plotted each BER point after accumulating more than 5,000 bit errors. 


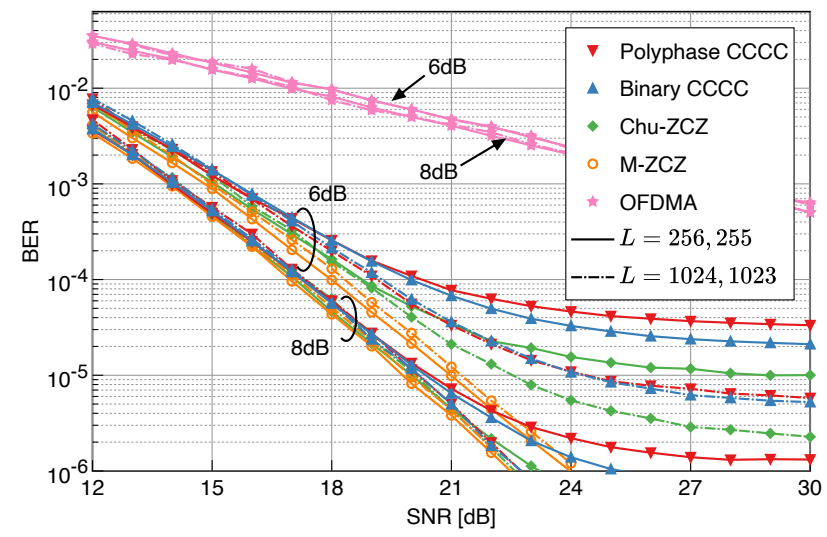

Fig. 6. Clipping resistance for CCC-CDMA in 4paths fading channel

\section{A. Clipping resistances}

In Fig. 6, we plotted the signal to noise ratio (SNR) vs. bit error rate (BER) over the quasistatic Rayleigh fading channel for clipping levels $\mathrm{PAPR}_{0}=6 \mathrm{~dB}$ and $8 \mathrm{~dB}$. Assuming polar clipping at the transmitters, we replaced the signal $\boldsymbol{x}$ by $\boldsymbol{x}^{\prime}$ as

$$
x^{\prime}(\ell)= \begin{cases}x(\ell), & |x(\ell)|^{2} \leq E_{\mathrm{th}} \\ \frac{\sqrt{E_{\mathrm{th}}}}{|x(\ell)|}(\ell), & |x(\ell)|^{2}>E_{\mathrm{th}}\end{cases}
$$

where $E_{\text {th }}=\bar{E}_{\boldsymbol{x}} \times \mathrm{PAPR}_{0}$ is clipping threshold with the average transmission energy $\bar{E}_{\boldsymbol{x}}=E_{\boldsymbol{x}} / L$ and detected the transmitted symbols based on the ML criterion with the perfect CIR at all receivers.

We can see from Fig. 6 that, as indicated in [24], the best performances are achieved by MZCZ for all cases and their BER curves exhibit the full path diversity without any error floors. On the other hand, OFDMA shows the worst performance for all cases due to it is lacking in diversity order while error floors are appeared in the BER curves of CCCCs and Chu-ZCZ. Fig. 6 also indicates that the lengthening employed sequence i.e., increment of SF is a simple but efficient countermeasure to mitigate the influence of clipping noise.

To explain the effect of SF, in Fig. 7, we plotted $\operatorname{Pr}\left\{\mathrm{PAPR}>\mathrm{PAPR}_{0}\right\}$, the complementary cumulative probability function $(\mathrm{CCPF})$ of

$$
\text { PAPR }:=\max _{\ell=0}^{L-1}\left\{|x(\ell)|^{2}\right\} / \bar{E}_{\boldsymbol{x}}
$$

after testing $10^{8}$ packets. We can see from this figure that, the CCPF curves of the polyphase 


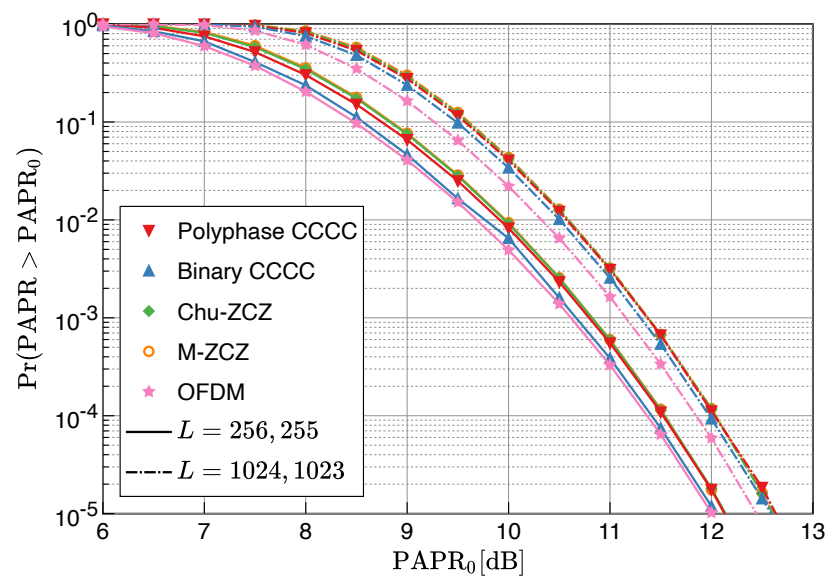

Fig. 7. PAPR distribution of CS-CDMA with Z-CCCs and MM

CCCC-ZCZ, Chu-ZCZ and MM-ZCZ with the same length are overlapped each other and hence the occurrence probabilities of them are almost the same. Thus, the different tolerances are caused by the difference in distribution of the despread clipping noise and it has the minimum energy for the white Gaussian distribution. Thus, M-ZCZ constructed from a PR sequence has best resistance, and despite the enlargement of SF increases the occurrence probability of clipping, the resistance against clipping noise is enhanced with a large SF since for these cases the distribution of the despread clipping noise is close to the white Gaussian and we may enjoy processing gain. Comparing the Chu-ZCZ of the same length, although CCCC has higher error floor level, the low complexity implementation of CCC-CDMA allows easy enlargement of SF to combat clipping noise.

\section{B. Doppler resistance}

Assuming carrier frequency of $2 \mathrm{GHz}$ and symbol duration $T_{s}=100 \mu \mathrm{s}$, in Fig. 8, we compared BER performances over the fast fading channels with the normalized maximum Doppler frequencies $f_{\max } T_{s}=0.04,0.01$, corresponding to moving velocities $f_{\max } T_{s}$ are $216 \mathrm{~km} / \mathrm{h}, 54$ $\mathrm{km} / \mathrm{h}$, respectively. The time varying channel is generated based on Jakes model [37] and we used the Rayleigh fading simulation model given in [38]. At the receiver, the ML detection is utilized with imperfect CIR information, i.e., the CIR at each begin of packet is used for the ML detection. 


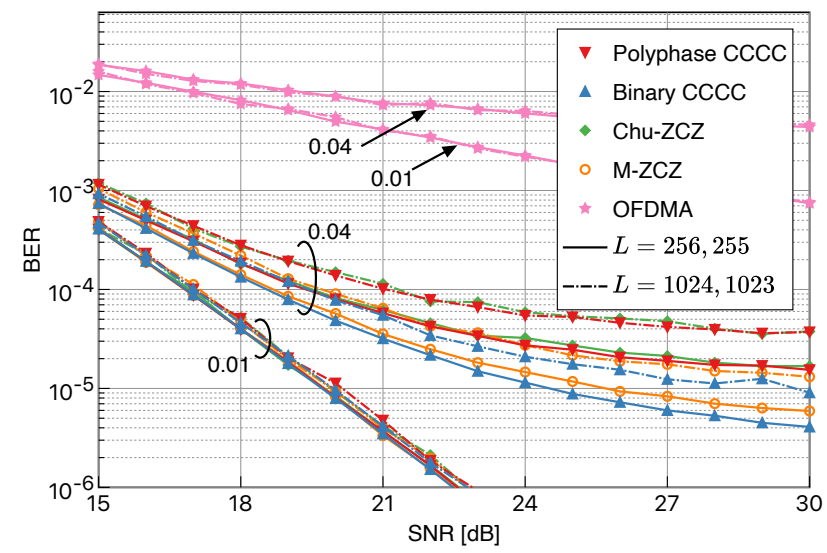

Fig. 8. Doppler resistance for CS-CDMA

Comparing the curves of the case $f_{\max } T_{s}=0.04$, the binary CCCC is more resistant than M-ZCZ against fast fading and the polyphase sequences show the similar weakness. In fact, over fast fading channel, the M-ZCZ and binary CCCC disperse ICIs over all users with similar strengths while the polyphase sequences concentrate them on few neighboring channels. Thus, for the polyphase sequences, we may mitigate the ICI caused over fast fading channels by a sophisticatedly designed channel estimation and equalization.

\section{CONCLUSION}

This paper presented CCC-CDMA with its comprehensive FT based implementation structure of transceiver to reduce the computational complexity of CS operation. Simulation results shown that the enlargement of SF strengthen the clipping resistance and the binary CCC-CDMA has excellent robustness against Doppler frequency shifts.

\section{ACKNOWLEDGMENT}

The author would like to thank Bohulu Kwame Ackah for his help and support in making this paper. This work was supported by JSPS KAKENHI Grant Numbers JP16K06339. 


\section{APPENDIX}

A. The proofs of (3) and (4)

The $\tau$ th entry of the left-hand side of (3) can be calculated as

$$
\begin{aligned}
\psi_{\mathrm{A}}\left(\boldsymbol{s}, \boldsymbol{\psi}_{\mathrm{A}}\left(\boldsymbol{s}^{\prime}, \boldsymbol{s}^{\prime \prime}\right) ; \tau\right) & =\sum_{\ell=0}^{L-1} s(\ell) \psi_{\mathrm{A}}\left(\boldsymbol{s}^{\prime}, \boldsymbol{s}^{\prime \prime} ; \tau-\ell\right) \\
& =\sum_{\ell=0}^{L-1} \sum_{\ell^{\prime}=0}^{L^{\prime}-1} s(\ell) s^{\prime}\left(\ell^{\prime}\right) s^{\prime \prime}\left(\tau-\ell-\ell^{\prime}\right)
\end{aligned}
$$

and it coincides with that of the right-hand side given by

$$
\begin{aligned}
\psi_{\mathrm{A}}\left(\boldsymbol{\psi}_{\mathrm{A}}\left(\boldsymbol{s}, \boldsymbol{s}^{\prime}\right), \boldsymbol{s}^{\prime \prime} ; \tau\right) & =\sum_{\substack{k=0 \\
L-1}}^{L+L^{\prime}-2} \psi_{\mathrm{A}}\left(\boldsymbol{s}, \boldsymbol{s}^{\prime} ; k\right) s^{\prime \prime}(\tau-k) \\
& =\sum_{\ell=0}^{L+L^{\prime}-2} \sum_{k=0}^{L-1} s(\ell) s^{\prime}(k-\ell) s^{\prime \prime}(\tau-k) \\
& =\sum_{\ell=0}^{L} \sum_{\ell^{\prime}=0}^{L^{\prime}-1} s(\ell) s^{\prime}\left(\ell^{\prime}\right) s^{\prime \prime}\left(\tau-\ell-\ell^{\prime}\right)
\end{aligned}
$$

where we let $k=\ell+\ell^{\prime}$ and the fact $s^{\prime}\left(\ell^{\prime}\right)=0$ for $\ell \geq L^{\prime}$ is utilized in the last equation.

In the same way, the $\tau$ th entries of the left- and right-hand sides of (4) are given by

$$
\begin{aligned}
\psi_{\mathrm{A}}\left(\overline{\boldsymbol{s}}, \underline{\phi}_{\mathrm{A}}\left(\boldsymbol{s}^{\prime}, \boldsymbol{s}^{\prime \prime}\right) ; \tau\right) & \left.=\sum_{\ell=0}^{L-1} s(\ell) \underline{\phi}_{\mathrm{A}}\left(\boldsymbol{s}^{\prime}, \boldsymbol{s}^{\prime \prime} ; \tau-\ell\right)\right] \\
& \left.=\sum_{\ell=0}^{L-1} s(\ell) \phi_{\mathrm{A}}\left(\boldsymbol{s}^{\prime}, \boldsymbol{s}^{\prime \prime} ; 1-L^{\prime \prime}-\tau+\ell\right)\right] \\
& =\sum_{\ell=0}^{L-1} \sum_{\ell^{\prime}=0}^{L^{\prime}-1} s(\ell) s^{\prime}\left(\ell^{\prime}\right)\left[s^{\prime \prime}\left(1-L^{\prime \prime}-\tau+\ell+\ell^{\prime}\right)\right]^{*}
\end{aligned}
$$

and

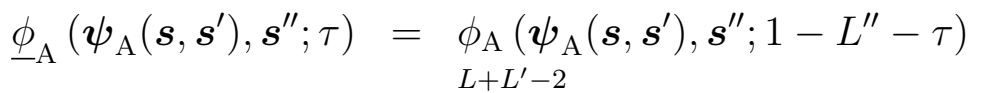

$$
\begin{aligned}
& =\sum_{k=0}^{L+L^{\prime}-2} \psi_{\mathrm{A}}\left(\boldsymbol{s}, \boldsymbol{s}^{\prime} ; k\right)\left[s^{\prime \prime}\left(1-L^{\prime \prime}-\tau+k\right)\right]^{*} \\
& =\sum_{\ell=0}^{L-1} \sum_{k=0}^{L+L^{\prime}-2} s(\ell) s^{\prime}(k-\ell)\left[s^{\prime \prime}\left(1-L^{\prime \prime}-\tau+k\right]^{*}\right. \\
& =\sum_{\ell=0}^{L-1} \sum_{\ell^{\prime}=0}^{L^{\prime}-1} s(\ell) s^{\prime}\left(\ell^{\prime}\right)\left[s^{\prime \prime}\left(1-L^{\prime \prime}-\tau+\ell+\ell^{\prime}\right)\right]^{*}
\end{aligned}
$$

respectively. Thus, the equality (4) holds. 


\section{B. Input-output relationship of CC-CDMA}

Since the equation (15) can be expressed as

$$
\begin{aligned}
\underline{\boldsymbol{r}}^{m} & =\sum_{n=0}^{N-1} \underline{\boldsymbol{\phi}}_{\mathrm{A}}\left(\boldsymbol{y}_{n}, \boldsymbol{c}_{n}^{m}\right) \\
& =\sum_{n=0}^{N-1} \underline{\boldsymbol{\phi}}_{\mathrm{A}}\left(\boldsymbol{\psi}_{\mathrm{A}}\left(\boldsymbol{h}_{P}, \boldsymbol{x}_{n}\right), \boldsymbol{c}_{n}^{m}\right)+\underline{\boldsymbol{\eta}}^{m}
\end{aligned}
$$

where $\underline{\boldsymbol{\eta}}^{m}=\sum_{n=0}^{N-1} \underline{\boldsymbol{\phi}}_{\mathrm{A}}\left(\boldsymbol{\xi}_{n}, \boldsymbol{c}_{n}^{m}\right)$. Thus, from (4), it can be further calculated as

$$
\begin{aligned}
\underline{\boldsymbol{r}}^{m} & =\sum_{n=0}^{N-1} \boldsymbol{\psi}_{\mathrm{A}}\left(\boldsymbol{h}_{P}, \underline{\boldsymbol{\phi}}_{\mathrm{A}}\left(\boldsymbol{x}_{n}, \boldsymbol{c}_{n}^{m}\right)\right)+\underline{\boldsymbol{\eta}}^{m} \\
& =\boldsymbol{\psi}_{\mathrm{A}}\left(\boldsymbol{h}_{P}, \sum_{n=0}^{N-1} \underline{\boldsymbol{\phi}}_{\mathrm{A}}\left(\boldsymbol{x}_{n}, \boldsymbol{c}_{n}^{m}\right)\right)+\underline{\boldsymbol{\eta}}^{m} \\
& =\boldsymbol{\psi}_{\mathrm{A}}\left(\boldsymbol{h}_{P}, \sum_{n=0}^{N-1} \underline{\boldsymbol{\phi}}_{\mathrm{A}}\left(\sum_{m^{\prime}=0}^{M-1} \boldsymbol{x}_{n}^{m^{\prime}}, \boldsymbol{c}_{n}^{m}\right)\right)+\underline{\boldsymbol{\eta}}^{m} \\
& =\boldsymbol{\psi}_{\mathrm{A}}\left(\boldsymbol{h}_{P}, \sum_{n=0}^{N-1} \underline{\boldsymbol{\phi}}_{\mathrm{A}}\left(\sum_{m^{\prime}=0}^{M-1} \boldsymbol{\psi}_{\mathrm{A}}\left(\boldsymbol{u}^{m^{\prime}}, \boldsymbol{c}_{n}^{m^{\prime}}\right), \boldsymbol{c}_{n}^{m}\right)\right)+\underline{\boldsymbol{\eta}}^{m} \\
& =\boldsymbol{\psi}_{\mathrm{A}}\left(\boldsymbol{h}_{P}, \sum_{m^{\prime}=0}^{M-1} \boldsymbol{\psi}_{\mathrm{A}}\left(\boldsymbol{u}^{m^{\prime}}, \sum_{n=0}^{N-1} \underline{\boldsymbol{\phi}}_{\mathrm{A}}\left(\boldsymbol{c}_{n}^{m^{\prime}}, \boldsymbol{c}_{n}^{m}\right)\right)\right)+\underline{\boldsymbol{\eta}}^{m}
\end{aligned}
$$

where the property (4) is utilized in the last equation and we notice that

$$
\sum_{n=0}^{N-1} \underline{\phi}_{\mathrm{A}}\left(\boldsymbol{c}_{n}^{m^{\prime}}, \boldsymbol{c}_{n}^{m} ; \tau\right)=L N \delta\left(m-m^{\prime}\right) \delta(-\tau-L+1)
$$

holds from (11). Thus, if we let $\boldsymbol{r}^{m}=\left(\underline{r}^{(m)}(\tau)\right)_{\tau=L-1}^{K+P+L-2}$, the output can be expressed as

$$
\boldsymbol{r}^{m}=L N \boldsymbol{\psi}_{\mathrm{A}}\left(\boldsymbol{h}_{P}, \boldsymbol{u}^{m}\right)+\boldsymbol{\eta}^{m}
$$


where $\boldsymbol{\eta}=\left(\underline{\eta}^{(m)}(\tau)\right)_{\tau=L-1}^{K+P+L-2}$ is CS Gaussian random vector with mean zero and covariance

$$
\begin{aligned}
E\left\{\boldsymbol{\eta}^{H} \boldsymbol{\eta}\right\} & =E\left\{\sum_{n=0}^{N-1} \sum_{n^{\prime}=0}^{N-1} \underline{\phi}_{\mathrm{A}}^{H}\left(\boldsymbol{\xi}_{n}, \boldsymbol{c}_{n}^{m}\right) \underline{\boldsymbol{\phi}}_{\mathrm{A}}\left(\boldsymbol{\xi}_{n^{\prime}}, \boldsymbol{c}_{n^{\prime}}^{m}\right)\right\} \\
& =\left[\sum_{n, n^{\prime}=0}^{N-1} \sum_{\ell, \ell^{\prime}=0}^{K+L+G-2} E\left\{\xi_{n}^{*}(\ell) \xi_{n^{\prime}}\left(\ell^{\prime}\right)\right\} c_{n}^{m}(-\tau+\ell)\left[c_{n^{\prime}}^{m}\left(-\tau^{\prime}+\ell^{\prime}\right)\right]^{*}\right]_{\tau, \tau^{\prime}=0}^{K+P-1} \\
& =\sigma^{2}\left[\sum_{n=0}^{N-1} \sum_{\ell=0}^{K+L+G-2} c_{n}^{m}(-\tau+\ell)\left[c_{n}^{m}\left(-\tau^{\prime}+\ell\right)\right]_{\tau, \tau^{\prime}=0}^{K+P-1}\right. \\
& =\sigma^{2}\left[\sum_{n=0}^{N-1} \phi_{\mathrm{A}}\left(\boldsymbol{c}_{n}^{m}, \boldsymbol{c}_{n}^{m} ; \tau-\tau^{\prime}\right)\right]_{\tau, \tau^{\prime}=0}^{K+P-1} \\
& =N L \sigma^{2} \boldsymbol{I}_{K+P}
\end{aligned}
$$

\section{Input-output relationship of CS-CDMA}

Since the CP removed signal can be expressed as

$$
\boldsymbol{y}=\boldsymbol{\psi}_{\mathrm{P}}(\boldsymbol{h}, \boldsymbol{x})+\boldsymbol{\xi}
$$

for $\boldsymbol{h}=\left(\boldsymbol{h}_{P}, \mathbf{0}_{L-P}\right)$ and $\boldsymbol{\xi}=(\vec{\xi}(\ell))_{\ell=G}^{G+L-1}$, the despread signal is given by

$$
\begin{aligned}
\underline{\boldsymbol{r}}^{m} & =\underline{\boldsymbol{\phi}}_{\mathrm{P}}\left(\boldsymbol{y}, \boldsymbol{s}^{m}\right) \\
& =\underline{\boldsymbol{\phi}}_{\mathrm{P}}\left(\boldsymbol{\psi}_{\mathrm{P}}(\boldsymbol{h}, \boldsymbol{x}), \boldsymbol{s}^{m}\right)+\underline{\boldsymbol{\eta}}^{m} \\
& =\boldsymbol{\psi}_{\mathrm{P}}\left(\boldsymbol{h}, \underline{\boldsymbol{\phi}}_{\mathrm{P}}\left(1 \sum_{m^{\prime}=0}^{M-1} \boldsymbol{\psi}_{\mathrm{P}}\left(\boldsymbol{d}^{m^{\prime}}, \boldsymbol{s}^{m^{\prime}}\right), \boldsymbol{s}^{m}\right)\right)+\underline{\boldsymbol{\eta}}^{m} \\
& =\boldsymbol{\psi}_{\mathrm{P}}\left(\boldsymbol{h}, \sum_{m^{\prime}=0}^{M-1} \boldsymbol{\psi}_{\mathrm{P}}\left(\boldsymbol{d}^{m^{\prime}}, \underline{\boldsymbol{\phi}}_{\mathrm{P}}\left(\boldsymbol{s}^{m^{\prime}}, \boldsymbol{s}^{m}\right)\right)\right)+\underline{\boldsymbol{\eta}}^{m}
\end{aligned}
$$

where $\boldsymbol{\eta}^{m}=\underline{\phi}_{\mathrm{P}}\left(\boldsymbol{\xi}, \boldsymbol{s}^{m}\right)$.

Let $\boldsymbol{r}^{m}=\left(\underline{r}^{(m)}(\ell)\right)_{\ell=0}^{K+P-1}$, for $K+P \leq Z$. Then, we have from (9) that

$$
\begin{aligned}
\boldsymbol{r}^{m} & =E_{\boldsymbol{s}^{m}}\left(\psi_{\mathrm{P}}\left(\boldsymbol{h}, \boldsymbol{d}^{m} ; \tau\right)\right)_{\tau=0}^{K+P-1}+\boldsymbol{\eta}^{m} \\
& =E_{\boldsymbol{s}^{m}} \boldsymbol{\psi}_{\mathrm{A}}\left(\boldsymbol{h}_{P}, \boldsymbol{u}^{m}\right)+\boldsymbol{\eta}^{m}
\end{aligned}
$$


where $\boldsymbol{\eta}^{m}=\left(\underline{\eta}^{(m)}(\ell)\right)_{\ell=0}^{K+P-1}$ is CS Gaussian random vector with mean zero and covariance

$$
\begin{aligned}
E\left\{\boldsymbol{\eta}^{H} \boldsymbol{\eta}\right\} & =E\left\{\underline{\boldsymbol{\phi}}_{\mathrm{P}}^{H}\left(\boldsymbol{\xi}, \boldsymbol{s}^{m}\right) \underline{\boldsymbol{\phi}}_{\mathrm{P}}\left(\boldsymbol{\xi}, \boldsymbol{s}^{m}\right)\right\} \\
& =\left[\sum_{\ell, \ell^{\prime}=0}^{K+P-1} E\left\{\xi^{*}(\ell) \xi\left(\ell^{\prime}\right)\right\} s^{m}\left([-\tau+\ell]_{K+P}\right)\left[s^{m}\left(\left[-\tau^{\prime}+\ell^{\prime}\right]_{K+P}\right)\right]^{*}\right]_{\tau, \tau^{\prime}=0}^{K+P-1} \\
& =\sigma^{2}\left[\phi_{\mathrm{P}}\left(\boldsymbol{s}^{m}, \boldsymbol{s}^{m} ; \tau-\tau^{\prime}\right)\right]_{\tau, \tau^{\prime}=0}^{K+P-1} \\
& =E_{\boldsymbol{s}^{m}} \sigma^{2} \boldsymbol{I}_{K+P}
\end{aligned}
$$

\section{Signal representations of the CS-CDMA with CCCC}

Since the $m$ th CCCC-ZCZ is represented by

$$
s^{(m)}(\ell)=\sum_{n=0}^{N-1} c_{n}^{(m)}(\ell-n D)
$$

the transmitted signal can be expressed as

$$
\begin{aligned}
x^{(m)}(\tau) & =\psi_{\mathrm{A}}\left(\boldsymbol{d}^{m}, \boldsymbol{s}^{m} ; \tau\right) \\
& =\sum_{n=0}^{N-1} \sum_{t=0}^{N D-1} u^{(m)}\left([\tau-t]_{N D}\right) c_{n}^{(m)}(t-n D) \\
& =\sum_{n=0}^{N-1} \sum_{k=0}^{N-1} \sum_{\ell=0}^{D-1} u^{(m)}\left([\tau-\ell-k D]_{N D}\right) c_{n}^{(m)}(\ell+(k-n) D) \\
& =\sum_{n=0}^{N-1} \sum_{\ell=0}^{N-1} u^{(m)}\left([\tau-\ell-n D]_{N D}\right) c_{n}^{(m)}(\ell)
\end{aligned}
$$

where we let $t=k D+\ell$, for $0 \leq k<N$ and $0 \leq \ell<D$.

Similarly, the received signal can be expressed as

$$
\begin{aligned}
\underline{r}^{(m)}(\tau) & =\underline{\phi}_{\mathrm{P}}\left(\boldsymbol{y}, \boldsymbol{s}^{m} ; \tau\right) \\
& =\phi_{\mathrm{P}}\left(\boldsymbol{y}, \boldsymbol{s}^{m} ;-\tau\right) \\
& =\sum_{n=0}^{N-1} \sum_{t=0}^{N D-1} y\left([-\tau-t]_{N D}\right)\left[c_{n}^{(m)}(t)\right]^{*} \\
& =\sum_{k=0}^{N-1} \sum_{\ell=0}^{D-1} \sum_{n=0}^{N-1} y\left([-\tau-\ell-k D]_{N D}\right)\left[c_{n}^{(m)}(\ell+k D)\right]^{*}
\end{aligned}
$$

and, substituting the fact $c_{n}^{(m)}(\ell+k D)=\delta(k-n) c_{n}^{(m)}(\ell)$, it can be represented as

$$
\underline{r}^{(m)}(\tau)=\sum_{\ell=0}^{D-1} \sum_{n=0}^{N-1} y\left([-\tau-\ell-n D]_{N D}\right)\left[c_{n}^{(m)}(\ell)\right]^{*}
$$




\section{REFERENCES}

[1] R. V. Nee and R. Prasad, OFDM for Wireless Multimedia Communications. Artech House, 2000.

[2] LTE Envolved Universal Terrestrial Radio Access (E-UTRA): Multiplexing and Channel Coding, Third Generation Partnership Project (3GPP) Std., Jan. 2011.

[3] P. Z. Fan and M. Darnell, Sequence Design for Communications Applications. John Wiley \& Sons Inc., 1996.

[4] J. A. C. Bingham, "Multicarrier modulation for data transmission: An idea whose time has come," IEEE Commun. Mag., vol. 28, pp. 5-14, May 1990.

[5] A. N. Akansu, O. Duhamel, X. Liu, and M. de Courville, "Orthogonal transmultiplexers in communications: A review," IEEE Trans. Signal Process., vol. 46, no. 4, pp. 979-995, Apr. 1998.

[6] C. Han and T. Hashimoto, "Almost linear interleaver and its application in coded OFDM over Rayleigh fading channel," in Proc. IEEE Int. Symp. Personal, Indoor and Mobile Radio Commun. (PIMRC’13), London, U.K., Sep. 2013, pp. 687-692.

[7] N. Suehiro and M. Hatori, “ $N$-shift cross-orthogonal sequences,” IEEE Trans. Inf. Theory, vol. IT-34, no. 1, pp. 143-146, Jan. 1988.

[8] C. Han, N. Suehiro, and T. Hashimoto, "A systematic framework for the construction of optimal complete complementary codes," IEEE Trans. Inf. Theory, vol. 57, no. 9, pp. 6033-6042, Sep. 2011.

[9] N. Suehiro, "A signal design without co-channel interference for approximately synchronized cdma systems," IEEE J. Sel. Areas Commun., vol. 12, no. 5, pp. 837-841, Jun. 1994.

[10] C. Han, T. Hashimoto, and N. Suehiro, "A new construction method of zero-correlation zone sequences based on complete complementary codes," IEICE Trans. Found., vol. E91-A, no. 12, pp. 3698-3702, Dec. 2008.

[11] N. Suehiro and N. Kuroyanagi, "Multipath-tolerant binary signal design for approximately synchronized CDMA systems without co-channel interference using complete complementary codes," in Proc. IEEE Global Telecommun. Conf. (GlobeCom'98), Sydney, NSW, Nov. 1998, pp. 1356-1361.

[12] N. Suehiro, N. Kuroyanagi, T. Imoto, and S. Matsufuji, "Very efficient frequency usage system using convolutional spread time signals based on complete complementary code," in Proc. IEEE Int. Symp. Personal, Indoor and Mobile Radio Commun. (PIMRC2000), vol. 2, London, Sep. 2000, pp. 1567-1572.

[13] H.-H. Chen, J.-F. Yeh, and N. Suehiro, "A multicarrier CDMA architecture based on orthogonal complementary codes for new generations of wideband wireless communications," IEEE Commun. Mag., vol. 39, no. 10, pp. 126-135, Oct. 2001.

[14] C. Han, N. Suehiro, J. Chen, N. Kuroyanagi, and M. Nakamura, "Simulation of a parallel transmission system for multipath property to estimate pilot signals and additional chip-shifted information transmission signals," in Proc. IEEE Int. Workshop Signal Design and Its Applications in Commun. (IWSDA'01), Chengdu, China, Sep. 2001, pp. 107-116.

[15] H.-H. Chen, H.-W. Chiu, and M. Guizani, "Orthogonal complementary codes for interference-free CDMA technologies," IEEE Trans. Wireless Commun., vol. 13, no. 1, pp. 68-79, Feb. 2006.

[16] T. Kojima, A. Fujiwara, K. Yano, M. Aono, and N. Suehiro, "Comparison of the two signal design methods in the CDMA systems using complete complementary codes," IEICE Trans. Found., vol. E89-A, no. 9, pp. 2299-2306, Sep. 2006.

[17] P. Z. Fan, N. Suehiro, N. Kuroyanagi, and X. M. Deng, "Class of binary sequences with zero correlation zone," IEE Electron. Lett., vol. 35, no. 10, pp. 777-779, May 1999.

[18] P. Z. Fan and L. Hao, "Generalized orthogonal sequences and their application in synchronous cdma systems," IEICE Trans. Found., vol. E83-A, no. 11, pp. 2054-2069, Nov. 2000.

[19] N. S. Weerasinghe, "Convolutional spreading code-division multiple-access systems with cyclic prefix (CS-CDMA/CP) for downlink transmission,” Ph.D. dissertation, Univ. Electro-Communications, Mar. 2007.

[20] N. S. Weerasinghe and T. Hashimoto, "Convolutional spreading CDMA and comparison with the DS-CDMA with RAKE receiver," IEEE Trans. Commun., vol. 54, no. 11, pp. 1918-1922, Nov. 2006. 
[21] N. S. Weerasinghe, D. Chen, and T. Hashimoto, "Space-code CS-CDMA systems over MISO frequency-selective rayleigh fading channels," IEEE Trans. Wireless Commun., vol. 7, no. 3, pp. 769-773, Mar. 2008.

[22] L. Yue, N. S. Weerashinghe, C. Han, and T. Hashimoto, "Partial multiuser detection for CS-CDMA/CP over multipath channels," IEEE Trans. Commun., vol. 58, no. 8, pp. 2305-2313, Aug. 2010.

[23] S. Zhou, G. B. Giannakis, and C. L. Martret, "Chip-interleaved block-spread code division multiple access," IEEE Trans. Commun., vol. 50, no. 2, pp. 235-248, Feb. 2002.

[24] N. S. Weerasinghe, C. Han, and T. Hashimoto, "CS-CDMA/CP with ZCZ codes from an M-sequence and its performance for downlink transmission over a multipath fading channel," IEICE Trans. Found., vol. E90-A, no. 6, pp. 1204-1213, Jun. 2007.

[25] D. C. Chu, "Polyphase codes with good periodic correlation properties," IEEE Trans. Inf. Theory, vol. 19, no. 4, pp. 531-532, Jul. 1972.

[26] L. Yue, C. Han, N. S. Weerashinghe, and T. Hashimoto, "Performance of coded CS-CDMA/CP with M-ZCZ code over a fast fading channel,” IEICE Trans. Commun., vol. E93-B, no. 9, pp. 2381-2388, Sep. 2010.

[27] L. Yue, C. Han, and T. Hashimoto, "CS-CDMA/CP with the chu sequence: A bandwidth-efficient multi-access scheme for fast fading multipath channels," in Proc. IEEE Int. Conf. Commun. (ICC'11), Kyoto, Japan, Jun. 2011, pp. 5-9.

[28] L. Yue, "On the performance of convolutional spreading CDMA with cyclic prefix over fast fading channels," Ph.D. dissertation, Univ. Electro-Communications, Mar. 2011.

[29] C. Han and T. Hashimoto, "Z-connectable complete complementary code and its application in CDMA," in Proc. IEEE Int. Symp. Infor. Theory (ISIT'09), Seoul, Korea, Jun. 2009, pp. 438-442.

[30] C. Han, L. Yue, and T. Hashimoto, "FFT implementable CS-CDMA and its performance under polar clipping noise," in Proc. IEEE Int. Workshop Signal Design and Its Applications in Commun. (IWSDA'11), Chengdu, China, Oct. 2011, pp. $165-168$.

[31] M. Tanimoto, H. Sumiyoshi, and M. Komai, "Synchronous spread-spectrum multiplex communication system by using a modified M-sequence,” IEICE Trans. Commun. (in Japanese), vol. J67-B, no. 3, pp. 297-304, Mar. 1984.

[32] X. H. Tang, P. Z. Fan, and S. Matsufuji, "Lower bounds on correlation of spreading sequence set with low or zero correlation zone,” IEE Electron. Lett., vol. 36, no. 6, pp. 551-552, Mar. 2000.

[33] S.-M. Tseng and M. R. Bell, "Asynchronous multicarrier DS-CDMA using mutually orthogonal complementary sets of sequences," IEEE Trans. Commun., vol. 48, no. 1, pp. 53-59, Jan. 2000.

[34] L. Lu and V. K. Dubey, "Performance of a complete complementary code-based spread-time CDMA system in a fading channel," IEEE Trans. Veh. Commun., vol. 57, no. 1, pp. 250-259, Jan. 2008.

[35] J. W. Craig, "A new, simple and exact result for calculating the probability of error for two-dimensional signal constellations," in Proc. IEEE Military Commun. Conf. (MILCOM'91), vol. 2, McLean, VA, Nov. 1991, pp. 571-575.

[36] M. K. Simon and D. Divsalar, "Some new twists to problems involving the gaussian probability integral," IEEE Trans. Commun., vol. 46, no. 2, pp. 200-210, Feb. 1998.

[37] W. C. Jakes, Microwave Mobile Communications. Piscataway, NJ:IEEE Press, 1994.

[38] Y. Li and X. Huang, "The simulation of independent Rayleigh faders," IEEE Trans. Commun., vol. 50, no. 9, pp. 1503-1514, Sep. 2002. 